Anne Peters/Tilmann Altwicker, in: Jörg Philipp Terhechte (Hrsg.), Enyzklopädie des Europarechts, Band 3 (Baden-Baden: Nomos 2014)

\title{
§ 13 Die Verfahren beim EGMR Peters/Altwicker
}

Anne Peters/Tilmann Altwicker

Literatur:

Besson, Samantha, The Extraterritoriality of the European Convention on Human Rights: Why Human Rights Depend on Jurisdiction and What Jurisdiction Amounts to, LJIL 2012, 857; Breuer, Marten, Art. 46, in: Karpenstein, Ulrich/Mayer, Franz C. (Hg.), EMRK, München, 2012; Cremer, Hans-Joachim, Entscheidung und Entscheidungswirkung, in: Dörr, Oliver/Grote, Thilo/Marauhn, Rainer (Hg.), EMRK/GG, Tübingen 2. Auf. 2013, Kap. 32; Kadelbach, Stefan, Rechtsschutz durch den Europäischen Gerichtshof für Menschenrechte, in: Ehlers, Dirk/Schoch, Friedrich (Hg.), Rechtsschutz im Öffentlichen Recht, Berlin, 2009, § 5; Frowein, Jochen A., The Binding Force of ECHR Judgments and its Limits, in: Breitenmoser, Stephan (Hg.), Human Rights, Democracy and the Rule of Law, in: FS Wildhaber, Zürich u.a., 2007, 261; Garlicki, Lech, Broniowski and After, in: Caflisch, Lucius (Hg)., Liber amicorum Luzius Wildhaber, Kehl u.a. 2007, 177; Grabenwarter, Christoph, Grundrechtsvielfalt und Grundrechtskonflikte im europäischen Mehrebenensystem. Wirkungen von EGMR-Urteilen und der Beurteilungsspielraum der Mitgliedstaaten, EuGRZ 2011, 229; ders., Wirkungen eines Urteils des Europäischen Gerichtshofs für Menschenrechte, JZ 2010, 857; Grewe, Constance, Beitritt der EU zur EMRK und ZP 14: Wirksame Durchsetzung einer gesamteuropäischen Grundrechteverfassung? EuR 2012, 285; Häberle, Peter, Europäische Verfassungslehre, 7. Aufl., Baden-Baden, 2011 ; Haider, Dominik, The Pilot-Judgment Procedure of the European Court of Human Rights, Leiden u.a., 2013; Keller, Helen/Forowicz, Magdalena/Engi, Lorenz, Friendly Settlements Before the European Court of Human Rights, Oxford, 2010; Klein, Eckart, Der Schutz der Grund- und Menschenrechte durch den Europäischen Gerichtshof für Menschenrechte, in: Merten, Detlef/Papier, Hand-Jürgen, Handbuch der Grundrechte in Deutschland und Europa VI/1, Heidelberg u.a., 2010, § 150; Payandeh, Mehrdad, Konventionswidrige Gesetze vor deutschen Gerichten, DÖV 2011, 382; Peters, Anne, Die Anwendbarkeit der EMRK in Zeiten komplexer Hoheitsgewalt und das Prinzip der Grundrechtstoleranz, AVR 2010, 1; dies./Altwicker, Tilmann, Europäische Menschenrechtskonvention, 2. Aufl., München, 2012; Schmahl, Stefanie, Pilot-Urteile als Mittel der Verfahrensbeschleunigung beim EGMR, EuGRZ 2008, 369; Stone Sweet, Alec, Sur la Constitutionnalisation de la Convention Européenne des Droits de 1'Homme, RTDH 2009, 923;Tomuschat, Christian, Individueller Rechtsschutz: das Herzstück des „ordre public européen“ nach der Europäischen Menschenrechtskonvention, EuGRZ 2003, 95; ders.,Die Erklärung von Brighton, SZIER 2012, 191; Wenzel, Nicola, Art. 41, in: Karpenstein, Ulrich/Franz C. Mayer (Hg.), EMRK, München, 2012; Wildhaber, Luzius, Eine verfassungsrechtliche Zukunft für den Europäischen Gerichtshof für Menschenrechte?, EuGRZ 2002, 569. 
Anne Peters/Tilmann Altwicker, in: Jörg Philipp Terhechte (Hrsg.), Enyzklopädie des Europarechts, Band 3 (Baden-Baden: Nomos 2014)

\section{A. Einleitung}

1

Der Europäische Menschenrechtsgerichtshof (EGMR) wurde 1953 eingerichtet und hat seinen Sitz in Straßburg. Das erste Urteil erging 1960. ${ }^{1}$ Seit $1998^{2}$ ist die Gerichtsbarkeit obligatorisch, d.h. jeder Mitgliedstaat der EMRK muss automatisch Beschwerden, die beim Gerichtshof gegen ihn eingelegt werden, ohne eine gesonderte Zustimmung zulassen. Seitdem ist der EGMR ein ständiger Gerichtshof (Art. 19 S. 2 EMRK); die Europäische Kommission für Menschenrechte wurde abgeschafft. Wegen der immensen Arbeitsbelastung des EGMR sieht das 14. Prot. (in Kraft seit 1.7.2010) wichtige Entlastungen vor. Das Protokoll hat eine zweifache Stoßrichtung: die schnellere Zurückweisung unzulässiger Beschwerden und die zügige Bewältigung repetitiver Fälle. Die hauptamtlichen Richter (einer pro Konventionsstaat) werden von der parlamentarischen Versammlung des Europarats für eine Amtszeit von neun Jahren gewählt (Art. 22 u. 23 EMRK).

\section{2}

Aufgabe des EGMR ist es, die Einhaltung der Verpflichtungen sicherzustellen, welche die Vertragsstaaten mit der Konvention eingegangen sind (Art. 19 EMRK). Im Rechtsschutzsystem der EMRK gibt es drei Verfahrensarten: Die Individualbeschwerde gem. Art. 34 EMRK, die Staatenbeschwerde gem. Art. 33 EMRK sowie das Gutachtenverfahren gem. Art. 47 EMRK, das bislang eine untergeordnete Bedeutung einnimmt. Die wichtigste Verfahrensart vor dem EGMR ist die Individualbeschwerde. In den Beschwerdeverfahren kann der EGMR den betroffenen Vertragsstaat zudem zu vorläufigen Maßnahmen verpflichten (Regel 39 EGMR-VerfO).

\section{B. Das System des Konventionsrechtsschutzes}

\section{Individualbeschwerde}

\section{Allgemeines}

3

Artikel 34 EMRK verleiht Einzelpersonen, die der Jurisdiktion eines Konventionsstaates unterstehen, ein individuelles, verfahrensbezogenes Recht auf effektive Ausübung der Beschwerdemöglichkeit. ${ }^{3}$ Diese Norm enthält nicht nur wesentliche Voraussetzungen der Zulässigkeit von Beschwerden, sondern auch ein subjektives Verfahrensgrundrecht, das den Vertragsstaaten untersagt, die wirksame Ausübung des Individualbeschwerderechts zu behindern (vgl. Art. 34 S. 2 EMRK). Positiv gewendet wird dem Einzelnen damit das Recht gewährleistet, frei mit dem Gerichtshof kommunizieren zu können, ohne einem direkten oder in-

* Dieser Beitrag basiert auf Peters/Altwicker, Europäische Menschenrechtskonvention, §§ 1, 35-37 in aktualisierter Form. Wir danken dem Verlag C.H.Beck für die freundliche Genehmigung der Verwendung unseres Textes.

${ }^{1}$ EGMR, 14.11.1960, Nr. 332/57 (Lawless/Irland).

${ }^{2}$ Inkrafttreten des 11. Protokolls und Ablauf einer einjährigen Übergangszeit.

${ }^{3}$ EGMR, 4.2.2005, Nr. $46827 / 99$ u.a., Rn 102 (Mamatkulov u.a./Türkei), EuGRZ 2005, 357. 
Anne Peters/Tilmann Altwicker, in: Jörg Philipp Terhechte (Hrsg.), Enyzklopädie des Europarechts, Band 3 (Baden-Baden: Nomos 2014)

direkten Druck durch die Behörden ausgesetzt zu werden, die Beschwerde zurücknehmen oder inhaltlich verändern zu müssen. ${ }^{4}$ Die Voraussetzungen der Zulässigkeit für die Individualbeschwerde sind Art. 34,35 EMRK sowie der Verfahrensordnung des EGMR zu entnehmen. ${ }^{5}$ Der EGMR sollte alle Zulässigkeitsvoraussetzungen der EMRK von Amts wegen prüfen, da diese auch die Grenze der Zuständigkeit des Gerichtshofs selbst angeben. ${ }^{6}$

\section{Zeitliche Vereinbarkeit}

4

Die zeitliche Unvereinbarkeit (,,incompatibility“) gem. Art. 35 Abs. 3 EMRK liegt vor, wenn das gerügte Verhalten vor Inkrafttreten der EMRK (oder eines ihrer Zusatzprotokolle) für den Beschwerdegegner stattfand und nicht mehr andauert. ${ }^{7}$ Anknüpfungspunkt für die Vereinbarkeit ratione temporis ist - positiv gewendet - der Zeitpunkt der behaupteten Verletzungshandlung. Hintergrund ist der allgemeine Grundsatz der Nichtrückwirkung völkerrechtlicher Verträge (vgl. Art. 28 WVRK).

\section{3. Örtliche Vereinbarkeit}

5

Wenn die EMRK in örtlicher Hinsicht nicht anwendbar ist, liegt ,incompatibility ratione loci“ vor. Nach allgemeinem Völkerrecht ist die staatliche Hoheitsgewalt (Jurisdiktion) im Ausgangspunkt auf das Staatsgebiet bezogen, also territorial. Nur in Ausnahmefällen stellen staatliche Handlungen, die außerhalb des eigenen Territoriums vorgenommen werden bzw. rechtliche Wirkungen im Ausland entfalten, „Hoheitsausübung“ im Sinne von Art. 1 EMRK dar (sog. extraterritoriale Hoheitsrechtsausübung). Die Anwendbarkeit der Konvention ratione loci ist in fünf Ausnahmesituationen problematisch, die im Folgenden erörtert werden. Der EGMR hat die Frage der Jurisdiktion früher als Aspekt der örtlichen Vereinbarkeit meist im Rahmen der Zulässigkeit geprüft, in neueren Entscheidungen jedoch, unseres Erachtens zu Recht, wegen der Komplexität der Fragen in die Begründetheitsprüfung verschoben. ${ }^{8}$

\section{a) Innerstaatliches Verhalten mit Konsequenzen außerhalb des Staatsgebietes}

6

Eine staatliche Maßnahme, die Auswirkungen außerhalb des Staatsgebietes hat, kann eine Ausübung von Hoheitsgewalt i.S.v. Art. 1 EMRK darstellen. Die konventionsrechtlichen Schutzpflichten verbieten, dass

${ }^{4}$ EGMR, 8.7.2004, Nr. 48787/99 Rn 480 ff (Ilaşcu u.a./Moldawien u. Russland), NJW 2005, 1849; EGMR, 20.1.2011 (Gisayev/Russland), Nr. 14811/04, Rn 165 ff.

${ }^{5}$ EGMR-Verfahrensordnung (EGMR-VerfO, Rules of Court) v. 1.5.2012.

${ }^{6}$ Vgl. EGMR, 8.3.2006, Nr. 59532/00 Rn 67 (Blečić/Kroatien), NJW 2007, 347. Jedenfalls die Opfereigenschaft wird von Amts wegen geprüft, s. EGMR, 22.3.2012, Nr. 30078/06, Rn 79 (Markin/Russland).

${ }^{7} \mathrm{Zu}$ den Problemfällen der fortdauernden Verletzungshandlung bzw. der Fortwirkung einer abgeschlossenen Verletzungshandlung s. Peters/Altwicker, EMRK, § 35 Rn 6 m.w.N.

${ }^{8}$ EGMR, 7.7.2011, Nr. 55721/07, Rn 139 (Al-Skeini u.a./Vereinigtes Königreich), NJW 2012, 283. 
Anne Peters/Tilmann Altwicker, in: Jörg Philipp Terhechte (Hrsg.), Enyzklopädie des Europarechts, Band 3 (Baden-Baden: Nomos 2014)

der Vertragsstaat den Einzelnen ,sehenden Auges“ in sein Unheil schickt bzw. dass ein Handeln des Vertragsstaates die Konventionsrechtsausübung in einem Drittstaat unmöglich macht. Für die konventionsrechtliche Schutzpflicht ist es unerheblich, aus welcher Quelle die Gefahr stammt, die sich im Drittstaat aktualisiert (z.B. staatliche Folter, Naturkatastrophe oder geographische Umstände) . Im erstgenannten Fall geht es regelmäßig um die Auslieferung oder Ausweisung einer Person, der in einem Drittstaat Menschenrechtsverletzungen drohen (Soering-Konstellation). ${ }^{9}$ Der zweite Fall betrifft die Situation der auf fremdem Hoheitsgebiet eintretenden, konventionswidrigen Auswirkungen eines Einreiseverbots (NadaKonstellation). ${ }^{10}$ Streng genommen wird die EMRK hier nicht extraterritorial angewendet, weil Anknüpfungspunkt die Auslieferungs-, Ausweisungs- oder Einreiseverbotsentscheidung des Konventionsstaats sind, die in diesem Staat getroffen und vollzogen werden. Es treten lediglich die (konventionswidrigen) Folgen im Fremdstaat ein. Wenn nur staatliches Unterlassen (im Gegensatz zu aktivem Tun) Auswirkungen außerhalb des Staatsgebietes hat, wird es in der Regel an der erforderlichen hoheitlichen Verbindung zwischen dem Vertragsstaat und dem Beschwerdeführer fehlen. Beispielsweise konnte eine außerhalb Dänemarks wohnende Person, die sich in ihrer Religionsfreiheit durch den von ihr empfundenen blasphemischen Charakter von Mohamedkarikaturen beeinträchtigt fühlte, nicht geltend machen, schon aufgrund der Passivität Dänemarks gegenüber der Veröffentlichung dieser Karikaturen in Dänemark unter dänischer Hoheitsgewalt zu stehen. ${ }^{11}$

\section{b) Effektive Gebietskontrolle über ein Fremdgebiet durch einen Konventionsstaat}

\section{7}

Erforderlich für die örtliche Anwendbarkeit der EMRK ist eine „effektive Gesamtkontrolle“. ${ }^{12}$ Diese kann auch außerhalb des eigenen Staatsgebiets durch eine umfassende militärische Präsenz (Bsp.: Besetzung von Nordzypern durch die Türkei - Loizidou-Konstellation) ${ }^{13}$ oder durch eine von dem anderen Konventionsstaat abhängige örtliche Verwaltung ausgeübt werden (Bsp.: De facto-Abhängigkeit der Verwaltung in Transnistrien von Russland - Ilaşcu-Konstellation). ${ }^{14}$ In diesen Fällen untersteht auch das fremde Staatsgebiet der „Hoheitsgewalt“ des Konventionsstaates iSv Art. 1 EMRK, und die Konvention ist anwendbar. Wann die Kontrolle „effektiv“ ist, bestimmt sich nicht anhand rechtlicher, sondern anhand faktischer Indikatoren, so dass die Legalität einer Besatzung keine Rolle spielt. Maßgeblich ist vor allem die Stärke der militärischen Präsenz, ferner das Ausmaß des militärischen, wirtschaftlichen und politischen Einflusses auf

${ }^{9}$ EGMR, 7.8.1989, Nr. 14038/88, Rn 86 ff (Soering/Vereinigtes Königreich), NJW 1990, 2183. Aktuelle Fälle: EGMR, 17.1.2012, Nr. 8139/09 (Othman (Abu Qatada)/Vereinigtes Königreich); EGMR, 17.1.2012, Nr. 9146/07 u.a. (Harkins u. Edwards/Vereinigtes Königreich)..

${ }^{10}$ EGMR, 12.9.2012, Nr. 10593/08 (Nada/Schweiz), NJOZ 2013, 1183 (Problem der Hoheitsgewalt der Schweiz wird nicht thematisiert).

${ }^{11}$ EGMR, 11.12.2006, Nr. 5853/06 (El Mahi/Dänemark).

${ }^{12}$ EGMR, 8.7.2004, Nr. 48787/99, Rn 315 (Ilaşcu/Moldawien u. Russland); EGMR, 16.11.2004, Nr. 31821/96, Rn 70 (Issa/Türkei).

${ }^{13}$ EGMR, 23.31995, Nr. 15318/89, Rn 62 (Loizidou/Türkei), ÖJZ 1995, 629.

${ }^{14}$ EGMR, 8.7.2004, Nr. 48787/99, Rn 316, 392 (Ilaşcu/Moldawien u. Russland); jetzt auch EGMR, 15.11.2011, Nr. 23687/05 (Ivanțoc/Moldawien u. Russland). 
Anne Peters/Tilmann Altwicker, in: Jörg Philipp Terhechte (Hrsg.), Enyzklopädie des Europarechts, Band 3 (Baden-Baden: Nomos 2014)

die örtliche Verwaltung. ${ }^{15}$ Hoheitsgewalt in diesem Sinne kann auch außerhalb des ,espace juridique“ der Konvention ausgeübt werden. ${ }^{16}$ Deshalb konnte beispielsweise das Vereinigte Königreich im Jahr 2003 im von ihm besetzten Irak Hoheitsgewalt ausüben, auf welche die EMRK anwendbar war. Die hierin liegende Gefahr eines „Menschenrechtsimperialismus“ dürfte gering sein.

c) Extraterritoriale, militärische Intervention eines Konventionsstaates ohne effektive territoriale Kontrolle

In der Konstellation der extraterritorialen, militärischen Intervention ohne effektive territoriale Kontrolle scheidet eine Anwendung der Konvention ratione loci in der Regel aus. Leitfall ist Banković u. a.v. 17 Mitgliedstaaten (2001): ${ }^{17}$ Die EMRK war ratione loci nicht auf die Bombardierung Ex-Jugoslawiens durch Konventionsstaaten anwendbar. Es ist derzeit nicht klar, ob der EGMR an der Banković-Rechtsprechung auf Dauer festhalten wird. In einer Zulässigkeitsentscheidung im Fall Pad u.a. (2007) hat der Gerichtshof die mutmaßliche Tötung von Iranern durch türkische Soldaten im Nordwesten Irans vom Hubschrauber aus als jurisdiktionsbegründend angesehen. ${ }^{18}$ Hier muss die weitere Rechtsprechung abgewartet werden.

d) Extraterritoriales Handeln eines Konventionsstaates mit Autorität (Einzelkontrolle) über Personen oder Sachen

9

Beim extraterritorialen Handeln mit Autorität über Personen oder Sachen sind drei Fallgruppen zu unterscheiden: ${ }^{19}$ Erstens besteht Konventionsgebundenheit, wenn der Vertragsstaat - aufgrund Zustimmung, Einladung oder Duldung des Territorialstaates - alle oder einige der öffentlichen Befugnisse ausübt, die normalerweise dem Territorialstaat zufallen. ${ }^{20}$ Zweitens ist die Konvention anwendbar bei Maßnahmen des diplomatischen und konsularischen Personals eines Konventionsstaates im Ausland sowie auf Schiffen und Flugzeugen, die unter der Flagge oder dem Hoheitszeichen des Konventionsstaates registriert sind. ${ }^{21}$

${ }^{15}$ EGMR, 7.7.2011, Nr. 55721/07, Rn 139 (Al-Skeini u.a./Vereinigtes Königreich), NJW 2012, 283; EGMR, 19.10.2012, Nr. 43370/04 u.a., Rn 106 f. (Catan u.a. v. Moldawien u. Russland).

${ }^{16}$ Ebd., Rn 142.

${ }^{17}$ EGMR, 12.12.2001, Nr. $52207 / 99$ (Banković u.a./17 Mitgliedstaaten), NJW 2003, 413.

${ }^{18}$ EGMR, 28.6.2007, Nr. 60167/00, Rn 52 ff (Pad u.a./Türkei), (Beschwerde aber unzulässig wegen fehlender Rechtswegerschöpfung).

${ }^{19}$ So die Darstellung der Rechtsprechung in EGMR, 7.7.2011, Nr. 55721/07, Rn 133-137 (Al-Skeini u.a./Vereinigtes Königreich), unter der Überschrift „State agent authority and control““.

${ }^{20}$ Im Irak konnte die britische Präsenz nach Absetzung von Saddam Hussein phasenweise auf eine Einladung der irakischen Interimsregierung gestützt werden.

${ }^{21}$ EGMR, 23.2.2012, Nr. 27765/09 , Rn 70 ff (Hirsi Jamaa u.a./Italien), NVwZ 2012, 809. 
Anne Peters/Tilmann Altwicker, in: Jörg Philipp Terhechte (Hrsg.), Enyzklopädie des Europarechts, Band 3 (Baden-Baden: Nomos 2014)

10

Drittens begründet auch das Verhalten von Amtsträgern eines Konventionsstaates, die - auf fremdem Hoheitsgebiet, auf fremdgeflaggten Schiffen oder in Flugzeugen - Personen oder Sachen ihrer ausschließlichen faktischen Kontrolle unterwerfen, die Konventionsverantwortlichkeit. ${ }^{22}$ Es ist unerheblich, ob die staatlichen Amtswalter im Rahmen ihrer Befugnisse oder ultra vires handelten. ${ }^{23}$ Der vom EGMR in dieser Situation verwendete Test verlangt nur, dass Amtsträger Personen oder Sachen auf fremdem Territorium unter ihre Autorität und Kontrolle bringen. ${ }^{24}$ In diese Fallgruppe gehören das Aufbringen eines fremdgeflaggten Schiffes auf Hoher See durch Amtsträger eines Konventionsstaates ${ }^{25}$ sowie die Inhaftierung irakischer Staatsbürger in einem vom Vereinigten Königreich geführten Gefängnis im Irak. ${ }^{26}$

e) Prekäre Hoheitsgewalt im eigenen Staatsgebiet

11

Hier liegt keine Extraterritorialität vor, sondern die „effektive (Gesamt-)Kontrolle“ des Gebietsstaates selbst ist fraglich. Der EGMR hat in diesen Fällen prekärer Hoheitsgewalt bislang stets aus Gründen der Effektivität des Menschenrechtsschutzes die Konvention für anwendbar ratione loci gehalten. Beispiele sind die Fortdauer der Inhaftierung in dem de facto autonomen Gebiet Abchasien innerhalb Georgiens trotz Freispruchs durch den Obersten Gerichtshof des Gesamtstaates. ${ }^{27}$ Übt der Gebietsstaat keine effektive Gewalt mehr aus, trifft ihn die positive Pflicht, diplomatische, ökonomische oder juridische Maßnahmen zum Schutz der Konventionsrechte zu ergreifen. ${ }^{28}$

\section{Sachliche Vereinbarkeit}

12

Wenn die EMRK in sachlicher Hinsicht nicht anwendbar ist, liegt ,incompatibility ratione materiae“ vor. An der sachlichen Anwendbarkeit der EMRK fehlt es, wenn (1) die Verletzung eines Rechts gerügt wird, das von der EMRK überhaupt nicht gewährleistet wird (Beispiel: Recht auf politisches Asyl) ${ }^{29}$, (2) in keinen Schutzbereich eines Konventionsrechts fällt (Beispiel: Recht, eine bestimmte Minderheitensprache im

22 EGMR, 12.5.2005, Nr. 46221/99 (Öcalan/Türkei), NVwZ 2006, 1267; EGMR, 29.3.2010, Nr. 3394/03, Rn 65 (Medvedyev u.a./Frankreich), NJOZ 2011, 231.

${ }^{23}$ EGMR, 8.7.2004, Nr. 48787/99, Rn 319 (Ilaşcu/Moldawien u. Russland).

${ }^{24}$ EGMR, 16.11.2004, Nr. 31821/96, Rn 71 (Issa/Türkei) (,authority and control“).

${ }^{25}$ EGMR, 29.3.2010, Nr. 3394/03, Rn 65 (Medvedyev u.a./Frankreich).

${ }^{26}$ EGMR, 2.3.2010, Nr. 61498/08, Rn 57 (Al-Saadoon/Vereinigtes Königreich); EGMR, 7.7.2011, Nr. 27021/08, Rn 85 (Al-Jedda/Vereinigtes Königreich).

${ }^{27}$ EGMR, 8.4.2004, Nr. 71503/01, Rn 139 (Assanidze/Georgien), NJW 2005, 2207 (Vermutungsregel für Jurisdiktion auf dem eigenen Territorium).

${ }^{28}$ EGMR, 19.10.2012, Nr. 43370/04 u.a., Rn 109 f (Catan u.a. v. Moldawien u. Russland).

${ }^{29}$ EGMR, 4.2.2005, Nr. $46827 / 99$ u. 46951/99, Rn 66 (Mamatkulov u. Askarov/Türkei). 
Anne Peters/Tilmann Altwicker, in: Jörg Philipp Terhechte (Hrsg.), Enyzklopädie des Europarechts, Band 3 (Baden-Baden: Nomos 2014)

Parlament zu benutzen) ${ }^{30}$ oder (3) bei Bestehen eines (wirksamen und in der konkreten Situation einschlägigen) Vorbehalts gem. Art. 57 EMRK. ${ }^{31}$

\section{Beschwerdeführer (,persönliche“6 Vereinbarkeit)}

13

Unzulässigkeitsgründe, die mit der Person des Beschwerdeführers zusammenhängen, führen zur ,incompatibility ratione personae".

\section{a) Partei-, Prozess- und Postulationsfähigkeit}

14

Art. 34 erlaubt Individualbeschwerden ,,jeder natürlichen Person, nichtstaatlichen Organisation oder Personengruppe“. Die prozessuale Parteifähigkeit richtet sich nach der materiell-rechtlichen Konventionsrechtsberechtigung. ${ }^{32}$ Die Konventionsrechtsberechtigung endet mit dem Tod des Beschwerdeführers. In drei Fällen lässt der EGMR die Fortführung einer bereits eingelegten Beschwerde nach dem Tod des Beschwerdeführers dennoch zu: Erstens, wenn Erben oder nahe Verwandte ein berechtigtes Interesse daran haben. ${ }^{33}$ Davon ist auszugehen, wenn die behauptete Konventionsverletzung direkt auch die Rechtsstellung des Erben oder nahen Verwandten betrifft, ${ }^{34}$ aber auch bei einem moralischen Interesse daran, dass dem verstorbenen Beschwerdeführer nachträglich Gerechtigkeit widerfahre. ${ }^{35}$ In einer zweiten Konstellation hat der EGMR auch Dritten die Fortsetzung einer Beschwerde gestattet, wenn diese ein berechtigtes Interesse nachweisen und der Beschwerde eine Bedeutung über den Einzelfall hinaus zukommt. ${ }^{36}$ In einer seltenen, dritten Konstellation hält der EGMR das Beschwerdeverfahren aufrecht, wenn die Achtung der Menschenrechte dies erfordert (vgl. Art. 37 Abs. 1 S. 2 EMRK). ${ }^{37}$

15

Unter ,nichtstaatliche Organisationen“ fallen alle juristischen Personen und Vereinigungen, wenn sie auf Dauer angelegt und organisiert sind. Auch wenn sie nach dem jeweiligen nationalen Recht nicht rechtsfähig sind, können sie unter Umständen Träger von EMRK-Rechten (z.B. der Religionsfreiheit aus Art. 9 EMRK) sein und diese als Organisation geltend machen, sodass die Zulässigkeitsvoraussetzungen (nur) in Bezug auf die Organisation vorliegen müssen. Im Gegensatz dazu sind „Personengruppen ${ }^{66}$ Gruppierungen, die

${ }^{30}$ EGMR, 21.9.2010, Nr. 39426/06, Rn 1 (Birk-Levy/Frankreich).

${ }^{31}$ EGMR, 24.4.2007, Nr. 40412/98, Rn 61 (V./Finnland).

${ }^{32}$ Näher dazu s. Peters/Altwicker, EMRK, § 2 Rn 14 ff.

${ }^{33}$ EGMR, 21.1.2009, Nr. 8713/03, Rn 26 (Janus/Polen).

${ }^{34}$ EGMR, 13.7.2006, Nr. 17671/02, Rn 25 (Ressegatti/Schweiz).

${ }^{35}$ EGMR, 25.11.2008, Nr. 36919/02, Rn 29 (Armonienè/Litauen).

${ }^{36}$ Vgl. EGMR, 13.12.2000, Nr. 33071/96 (Malhous/Tschechische Republik).

${ }^{37}$ EGMR 23.7.2003, Nr. 40016/98, Rn 27 f. (Karner/Österreich), ÖJZ 2004, 36. 
Anne Peters/Tilmann Altwicker, in: Jörg Philipp Terhechte (Hrsg.), Enyzklopädie des Europarechts, Band 3 (Baden-Baden: Nomos 2014)

regelmäßig nur für vorübergehende Dauer bestehen und über einen sehr niedrigen Organisationsgrad verfügen (Bsp.: Bürgerinitiative). ${ }^{38}$ Bei Beschwerden einer „Personengruppe“ müssen alle Zulässigkeitsvoraussetzungen bei allen Mitgliedern erfüllt sein, denn es können nur Rechte der Mitglieder geltend gemacht werden. Nichtstaatliche Organisationen bzw. Personengruppen können Beschwerde allerdings nur soweit erheben, wie das Grundrecht auf sie passt, ansonsten sind sie bereits nicht parteifähig. Nicht parteifähig sind staatliche Organisationen bzw. juristische Personen des öffentlichen Rechts oder Stellen, die staatliche Funktionen ausüben. ${ }^{39}$

\section{6}

An die nicht explizit geregelte Prozessfähigkeit, d.h. die Fähigkeit, das Individualbeschwerdeverfahren selbst oder durch einen Bevollmächtigten zu führen, werden weniger strenge Anforderungen als in nationalen Prozessordnungen gestellt. Geschäftsunfähige und Minderjährige können unter Umständen selbst eine Individualbeschwerde einreichen. ${ }^{40}$

\section{7}

Davon zu trennen ist die Postulationsfähigkeit, d.h. die Fähigkeit, Verfahrenshandlungen die vorgeschriebene Form geben zu können. Nach Regel 36 EGMR-VerfO besteht zunächst für die Einreichung der Individualbeschwerde kein Anwaltszwang. Sobald allerdings die Beschwerde der gegnerischen Vertragspartei zugestellt wurde, muss sich der Beschwerdeführer anwaltlich vertreten lassen, da nur ein von einer Vertragspartei zugelassener Rechtsanwalt (,,advocate“) wirksame Prozesshandlungen vornehmen kann (Regel 36 Abs. 2 u. 4 EGMR-VerfO). Der Kammerpräsident kann Ausnahmen zulassen.

\section{b) Opfereigenschaft}

\section{8}

Der Beschwerdeführer muss geltend machen, Opfer einer Konventionsverletzung zu sein. Die Opfereigenschaft wird manchmal auch „Beschwer“ oder ,Sachlegitimation“ genannt und entspricht im deutschen Prozessrecht der „Beschwerdebefugnis“. Der Begriff des „Opfers“ enthält drei Elemente:

19

Der Beschwerdeführer muss erstens selbst betroffen sein. ${ }^{41}$ Es müssen eigene Rechte, nicht fremde Rechte oder Rechte der Allgemeinheit geltend gemacht werden. Das Erfordernis der Opfereigenschaft schließt in-

\footnotetext{
${ }^{38}$ Vgl. EGMR, 23.7.1968, Nr. 1474/62, Rn 2 (Belgischer Sprachenfall), EuGRZ 1975, 298.

${ }^{39}$ Zum Erfordernis der „Nichtstaatlichkeit“" s. Peters/Altwicker, EMRK, § 2 Rn 16.

${ }^{40}$ EGMR, 16.7.2009, Nr. 20082/02, Rn 39 (Zehentner/Österreich), ÖJZ 2010, 92. Mangels Prozessfähigkeit (locus standi) kann eine Individualbeschwerde ganz oder teilweise unzulässig sein, vgl. EGMR 27.4.2010, Nr. 16318/07, Rn 28 ff (Moretti u. Benedetti/Italien): fehlende Vertretungsbefugnis von Pflegeeltern eines minderjährigen Kindes.

${ }^{41}$ S. EGMR (GK), 7.6.2012, Nr. 38433/09, Rn 92 (Centro Europa 7 S.R.L. u. Di Stefano /Italien).
} 
Anne Peters/Tilmann Altwicker, in: Jörg Philipp Terhechte (Hrsg.), Enyzklopädie des Europarechts, Band 3 (Baden-Baden: Nomos 2014)

sofern die actio popularis aus. ${ }^{42}$ Jedoch kann bei Verletzung von Art. 2 oder 3 EMRK auch ein Vertreter für das Opfer klagen, selbst wenn er die Klage nicht im fremden Namen (als offengelegter Vertreter) erhebt. ${ }^{43}$ Eine andere Situation ist die, dass der Angehörige von Verschwundenen selbst als Opfer einer unmenschlichen und erniedrigenden Behandlung (Art. 3 EMRK) angesehen wird und seine Beeinträchtigung als Verletzung eines eigenen Rechts geltend macht (sog. mittelbare Opfereigenschaft). ${ }^{44}$ Dafür sind folgende Faktoren relevant: besondere Nähebeziehung (z.B. Eltern des Opfers), Grad der eigenen Involvierung in die Geschehnisse (z.B. wenn der Angehörige Zeuge der Ereignisse war oder versucht hat, Informationen über den Verbleib des Opfers zu erhalten) sowie schließlich die Art und Weise, wie die Behörden mit Anfragen der Angehörigen umgegangen sind. ${ }^{45}$

\section{0}

Die Opfereigenschaft bezieht sich zweitens auf den Zeitpunkt der Konventionsverletzung. Der Normalfall ist, dass eine gegenwärtige Verletzung gerügt wird. Eine vergangene (bereits abgeschlossene) Verletzung kann gerügt werden, wenn sie nicht geheilt wurde. Eine Heilung setzt voraus, dass die Vertragspartei die Konventionsverletzung zumindest der Sache nach anerkannt hat und Wiedergutmachung (z.B. Entschädigungszahlung, Rückübertragung von zu Unrecht enteignetem Grundstück) geleistet hat. ${ }^{46}$ Eine zukünftige Verletzung kann gerügt werden, wenn eine Rechtsverletzung nachweislich wahrscheinlich ist; eine bloße Vermutung reicht allerdings nicht aus. ${ }^{47}$ Es ist nicht zuzumuten, eine Verletzung abzuwarten. Die Opfereigenschaft besteht bereits, wenn eine Entscheidung ergeht, nicht erst bei ihrem Vollzug. Also reicht eine Ausweisungsverfügung, die Abschiebung muss nicht abgewartet werden.

21

Der dritte Aspekt der Opfereigenschaft ist die unmittelbare Betroffenheit. Diese liegt unproblematisch vor, wenn der Beschwerdeführer Adressat eines Verwaltungsakts oder eines Gerichtsurteils ist. Sie fehlt in der Regel bei der bloßen Betroffenheit durch ein Gesetz. Man muss erst den Vollzug abwarten. Hiervon werden aber mehrere Ausnahmen anerkannt. Das Abwarten einer behördlichen Vollzugsmaßnahme ist unzumutbar, wenn es sich um verdeckte Maßnahmen handelt, von denen der Beschwerdeführer möglicherweise nie Kenntnis erhalten wird. ${ }^{48}$ Eine weitere Ausnahme ist anerkannt für den Fall, dass das Gesetz den Behörden kein Ermessen bei der Anwendung lässt (z.B. bei Ladenschlusszeiten oder erbrechtlichen Rege-

42 Vgl. EGMR, 8.7.1980, Nr. 8727/79, J., B. und 361 (Eltern/Schweiz); vgl. auch EGMR, 25.11.1999, Nr. 46306/99 (Očić/Kroatien); EGMR, 15.3.2012, Nr. 4149/04 u.a., Rn 50 ff (Aksu/Türkei) (ausreichend für die Selbstbetroffenheit ist bereits die Betroffenheit als Mitglied einer ethnischen Gruppe).

${ }^{43}$ EGMR, 27.6.2000, Nr. 22277/93, Rn 49 ff (Ilhan/Türkei).

${ }^{44}$ EGMR, 13.6.2000, Nr. 23531/94, Rn 91 ff (Timurtas/Türkei).

${ }^{45}$ EGMR, 28.10.2010, Nr. 35079/04, Rn 121 (Sasita Israilova u.a./Russland).

${ }^{46}$ EGMR, 29.3.2006, Nr. 36813/97, Rn 180 (Scordino/Italien (Nr. 1)), NJW 2007, 1259.

${ }^{47}$ Vgl. EGMR, 6.11.2001, Nr. 53430/99 (The Christian Federation of Jehovah's Witnesses in France/Frankreich).

${ }^{48}$ EGMR, 6.9.1978, Nr. 5029/71, Rn 34 (Klass/Deutschland), EuGRZ 1979, 278 = NJW 1979, 1755. 
Anne Peters/Tilmann Altwicker, in: Jörg Philipp Terhechte (Hrsg.), Enyzklopädie des Europarechts, Band 3 (Baden-Baden: Nomos 2014)

lungen). ${ }^{49}$ Ebenso kann dem Beschwerdeführer nicht zugemutet werden, bei sanktionsbewehrten Normen (z.B. Strafnormen) erst die Verhängung einer Sanktion abzuwarten. ${ }^{50}$ In diesen Situationen kann (gegebenenfalls nach Erschöpfung des innerstaatlichen Rechtswegs) direkt gegen das Gesetz Beschwerde eingelegt werden.

\section{c) Erheblicher Nachteil}

\section{2}

Der Beschwerdeführer muss einen erheblichen Nachteil erlitten haben. Die Konvention drückt dies umgekehrt aus: Die Beschwerde ist unzulässig, wenn der Beschwerdeführer keinen erheblichen Nachteil erlitten hat, Art. 35 Abs. 3 lit. b) EMRK. Dieser Unzulässigkeitsgrund wurde mit dem 14. Protokoll (in Kraft seit dem 1.6.2010) eingeführt, um Beschwerden schneller abweisen zu können und so die Überlastung des Gerichtshofs abzumildern. ${ }^{51}$ Damit wird dem Gerichtshof die Kompetenz gegeben, „schwere“ von „weniger schweren“"Menschenrechtsverletzungen zu unterscheiden. Diese Möglichkeit entspricht eher der Vorstellung, dass der EGMR nicht unbedingt in jedem einzelnen Fall Hilfe gewähren muss, sondern in der Art eines „Verfassungsgerichts“ allgemein für die Aufrechterhaltung und Anhebung eines menschenrechtlichen Schutzniveaus zuständig sein soll (s. zu diesem Verfassungsgerichtsparadigma Rn 67). Wann ein erheblicher Nachteil vorliegt, muss ausgehend vom Einzelfall (v.a. der persönlichen Situation des Beschwerdeführers) ermittelt werden. Dabei spielen die subjektive Wahrnehmung des Nachteils durch den Beschwerdeführer sowie objektive Kriterien eine Rolle. ${ }^{52}$ Die Höhe eines erlittenen finanziellen Schadens ist oft relevant, ${ }^{53}$ aber nicht ausschlaggebend, wenn die Beschwerde menschenrechtliche Grundsatzfragen berührt. ${ }^{54}$ Beispielsweise warf ein Beschwerdeführer in einem Rechtsstreit um einen Kauf, in dem es um ca. 30.000 Euro ging, den tschechischen Gerichten Willkür vor. Das tschechische Verfassungsgericht holte von den Fachgerichten Stellungnahmen ein, gab dem Beschwerdeführer aber keine Gelegenheit zur Äußerung dazu. In Bezug auf ein faires Verfahren i.S.v. Art. 6 EMRK sah der EGMR hier keinen erheblichen Nachteil und erklärte die Beschwerde, da auch die Sicherheitsklauseln (dazu sogleich Rn23) kein Eintreten erforderten, für unzulässig. ${ }^{55}$

${ }^{49}$ EGMR, 29.4.2008, Nr. 13378/05, Rn 34 f. (Burden/Vereinigtes Königreich), NJW-RR 2009, 1606.

${ }^{50}$ EGMR, 26.10.1988, Nr. 10581/83, Rn 28 ff (Norris/Irland), EuGRZ 1992, 477.

${ }^{51}$ Vgl. Explanatory Report, Protokoll Nr. 14, CETS Nr. 194. Der neue Unzulässigkeitsgrund ist geleitet vom Grundsatz ,de minimis non curat praetor“.

52 EGMR, 6.3.2012, Nr. 23563/07, Rn 55 (Gagliano Giorgi/Italien)

${ }^{53}$ EGMR, 1.6.2010, Nr. 36659/04 (Ionescu/Rumänien) (finanzieller Schaden von ca. 90 Euro kein erheblicher Nachteil); EGMR, 21.12.2010, Nr. 45867/07 (Gaglione u.a./Italien) (finanzieller Schaden von mindestens 200 Euro ausreichend).

\footnotetext{
${ }^{54}$ Vgl. EGMR, 1.7.2010, Nr. 25551/05 (Korolev/Russland), NJW 2010, 3081.

${ }^{55}$ EGMR, 14.12.2010, Nr. 24880/05 (Holub/Tschechische Republik), NJW 2011, 3143.
} 
Anne Peters/Tilmann Altwicker, in: Jörg Philipp Terhechte (Hrsg.), Enyzklopädie des Europarechts, Band 3 (Baden-Baden: Nomos 2014)

23

Liegt kein erheblicher Nachteil vor, ist zu prüfen, ob eine der beiden Sicherheitsklauseln des Art. 35 Abs. 3 lit. b) EMRK greift, welche die Beschwerde doch zulässig machen. Die Beschwerde scheitert erstens nicht am neuen Unzulässigkeitsgrund, wenn ,,die Achtung der Menschenrechte ... eine Prüfung der Begründetheit“" „erfordert“ (ordre public-Klausel). ${ }^{56}$ Eine Prüfung ist erforderlich, wenn der Fall allgemeine Fragen zur Achtung der Konventionsrechte betrifft, wenn eine Verpflichtung der Vertragsstaaten klargestellt werden muss oder wenn der Staat einen Strukturmangel beheben muss. Die zweite Ausnahme liegt vor, wenn die Rechtssache „,noch von keinem innerstaatlichen Gericht gebührend geprüft“ wurde (Rechtsverweigerungsklausel). ${ }^{57}$ Wenn eine der beiden Sicherheitsklauseln eingreift, muss der Gerichtshof auf die Beschwerde trotz eines fehlenden erheblichen Nachteils des Beschwerdeführers eintreten.

\section{Beschwerdegegner}

24

Unzulässigkeitsgründe, die mit dem Beschwerdegegner (der Vertragspartei) zusammenhängen, führen ebenfalls zur „incompatibility ratione personae“. Die Parteieigenschaft des Beschwerdegegners fehlt (mit der Folge der Unzulässigkeit ratione personae), wenn die Beschwerde einen Staat als Beschwerdegegner benennt, der die Konvention ${ }^{58}$ oder das betreffende Zusatzprotokoll nicht ratifiziert ${ }^{59}$ hat, eine internationale oder supranationale Organisation als Beschwerdegegner benennt ${ }^{60}$ oder sich gegen ein Individuum richtet. ${ }^{61}$ Die behauptete Konventionsverletzung muss ferner der Vertragspartei zurechenbar sein. Bei der Frage der Zurechnung handelt es sich um ein Problem der Begründetheit, wie sich an dem systematischen Bezug zur materiell-rechtlichen Norm des Art. 1 EMRK zeigt. Der EGMR hat in früheren Entscheidungen die Zurechnung im Rahmen der Zulässigkeitsprüfung ratione personae behandelt, neuerdings aber auch im Rahmen der Begründetheit (,,merits“) geprüft. ${ }^{62}$ Letzteres ist in systematischer Hinsicht vorzugswürdig. ${ }^{63}$

${ }^{56}$ Hier kann der EGMR an seine Rechtsprechung zu dem ähnlichen Kriterium in Art. 37 Abs. 1 S. 2 EMRK (betreffend die Streichung von Beschwerden) anknüpfen.

${ }^{57}$ Das - noch nicht in Kraft getretene - 15. Protokoll sieht die Abschaffung der Rechtsverweigerungsklausel vor, s. 15. Protokoll, 24.6.2013 (ETS Nr. 213).

${ }^{58}$ EGMR, 28.8.1957, Nr. 262/57 (E.S./Deutschland).

${ }^{59}$ EGMR, 7.5.2013, Nr. 19840/09, Rn 125 (Shindler/Vereinigtes Königreich).

${ }^{60}$ EGMR, 11.12.2008, Nr. 45267/06 (Stephens/Zypern), Türkei und VN.

${ }^{61}$ EGMR, 18.9.2007, Nr. 55896/00 (Bakalov u.a./Bulgarien) (Insolvenzverwalter); EGMR, 19.10.2000, Nr. 45995/99 (Rutkowski/Polen) (Anwalt).

${ }^{62}$ EGMR, 7.7.2011, Nr. 27021/08, Rn 61 u. 80-84 (Al-Jedda/Vereinigtes Königreich).

${ }^{63}$ Wenn man der Zulässigkeitslösung des EGMR folgen will, wäre es richtiger, die Zurechnung unter dem Unzulässigkeitsgrund der „offensichtlichen Unbegründetheit“" zu prüfen. 
Anne Peters/Tilmann Altwicker, in: Jörg Philipp Terhechte (Hrsg.), Enyzklopädie des Europarechts, Band 3 (Baden-Baden: Nomos 2014)

\section{5}

Für Fragen der Zurechnung von Konventionsverletzungen sind die Grundsätze des Rechts der Staatenverantwortung einschlägig (vgl. Art. 4-11 ILC-Artikel zur Staatenverantwortung). ${ }^{64}$ Unproblematisch wird danach dem Vertragsstaat jegliches Handeln staatlicher Organe zugerechnet, auch ultra vires Handeln. ${ }^{65}$ Unter Umständen kann auch das Verhalten Privater zugerechnet werden, insbesondere wenn diese öffentliche Funktionen ausüben. $^{66}$

\section{6}

Für die Frage der Zurechnung von staatlichem Verhalten im Rahmen der EU oder internationaler Organisationen, insbesondere den Vereinten Nationen, hat die Rechtsprechung mehrere Grundprinzipien entwickelt. ${ }^{67}$ Grundsätzlich bleibt eine Auffangkonventionsbindung der Mitgliedstaaten bestehen. ${ }^{68}$ Voraussetzung der Konventionsbindung des Staates für Maßnahmen in Zusammenarbeit mit einer Organisation ist seine über die bloße Mitgliedschaft in der Organisation hinausgehende, „direkte oder indirekte“ Mitwirkung an der gerügten Beeinträchtigung. ${ }^{69}$ Für die Zurechnung und damit die Ausübung von „Hoheitsgewalt““ i.S.v. Art. 1 EMRK kommt es nach den völkerrechtlichen Grundsätzen ${ }^{70}$ auf die „effektive Kontrolle“ an. ${ }^{71}$ Ferner hat der EGMR eine widerlegliche „Vermutung“ aufgestellt, dass keine Verletzung der EMRK stattfand, wenn und solange die Organisation, in deren Rahmen der Vertragsstaat tätig geworden ist, ihrerseits

64 „Draft Articles on Responsibility of States for Internationally Wrongful Acts“ der International Law Commission, die von der UN-Generalversammlung zur Kenntnis genommen wurden, und die zum Großteil Gewohnheitsrecht widerspiegeln (UN Doc. A/56/10 (2001), S. 43 ff (nachfolgend ILC-Artikel).

${ }^{65}$ Vgl. EGMR, 8.7.2004, Nr. 48787/99, Rn 319 (Ilaşcu u.a./Moldau u. Russland).

${ }^{66}$ Vgl. dazu im Einzelnen Peters/Altwicker, EMRK, § 2 Rn 18 ff.

${ }^{67}$ Im Einzelnen ebd., § 2 Rn 22 ff.

${ }^{68}$ Sie bleiben sogar voll verantwortlich, wenn sie nur anlässlich der Mitgliedschaft in der Organisation freiwillige, gesonderte Maßnahmen ergreifen, vgl. EGMR, 18.2.1999, Nr. 24833/94 (Matthews/Vereinigtes Königreich), EuGRZ 1999, 200.

${ }^{69}$ EGMR, 9. 9. 2008, Nr. 73250/01 (Boivin/34 Mitgliedstaaten des Europarates).

${ }^{70}$ Draft Articles on the Responsibility of International Organisations („DARIO 2011“), Report of the ILC, 63d sess., GA OR 66, suppl. no. 10 (UN Doc. A/66/10), angenommen am 12.8.2011 (maßgeblich ist Art. $7)$.

${ }^{71}$ Ein Beispiel sind Internierungsmaßnahmen britischer Truppen im Irak im Jahr 2004 im Kontext der britischen Präsenz, die ihre Rechtsgrundlage unter anderem in UN-Sicherheitsratsresolutionen hatten. Diese waren dem Vereinigten Königreich zurechenbar. Sie lagen damit in der „Jurisdiktion“ des Vereinigten Königreichs i.S.v. Art. 1, und somit war das Vereinigte Königreich der richtige Beschwerdegegner, s. EGMR, 7.7.2011, Nr. 27021/08, Rn 74-86 (Al-Jedda/Vereinigtes Königreich). 
Anne Peters/Tilmann Altwicker, in: Jörg Philipp Terhechte (Hrsg.), Enyzklopädie des Europarechts, Band 3 (Baden-Baden: Nomos 2014)

die Grundrechte respektiert und einen ,äquivalenten“ Schutz bietet. ${ }^{72}$ Dies trifft auf die EU, nicht aber auf die UNO zu. ${ }^{73}$

\section{Erschöpfung des innerstaatlichen Rechtswegs (Art. 35 Abs. 1 EMRK)}

\section{a) Allgemeines}

27

Die Zulässigkeitsvoraussetzung der Rechtswegerschöpfung reflektiert den völkergewohnheitsrechtlichen Grundsatz der local remedies. ${ }^{74}$ Das prozessuale Erfordernis der Rechtswegerschöpfung ist mit dem Recht auf wirksame Beschwerde in Art. 13 EMRK verzahnt: Das Erfordernis der Rechtswegerschöpfung reicht nur so weit, wie die nationalen Rechtsbehelfe den Anforderungen des Art. 13 EMRK genügen. ${ }^{75}$

28

Zentraler Grundsatz ist die flexible und nicht-formalistische Anwendung des Art. 35 Abs. 1 EMRK. Das Erschöpfungserfordernis gilt weder absolut noch automatisch. Es müssen die Umstände des Einzelfalles und der gesamte Kontext des nationalen Rechtssystems beachtet werden. ${ }^{76}$ Grundsätzlich muss der Beschwerdeführer alle verfügbaren Rechtsbehelfe erschöpfen (institutionelle Rechtswegerschöpfung). Konkret verlangt Art. 35 Abs. 1 EMRK in Deutschland insbesondere, den Weg des Widerspruchsverfahrens zu gehen und Verfassungsbeschwerde beim BVerfG ${ }^{77}$ einzulegen. Der Beschwerdeführer muss daher zumindest eine Kopie des Nichtannahmebeschlusses des BVerfG nach Art. 93 b i.V.m. Art. 93 a BVerfGG vorlegen. Nach einem Strafverfahren ist kein Antrag auf Wiederaufnahme nötig, denn das Wiederaufnahmeverfahren gehört nicht zum ordentlichen Rechtsweg. Im Normalfall dürfte für die sinngemäße Rüge von Art. 6 EMRK bezüglich des Strafverfahrens aber eine Verfassungsbeschwerde möglich und erforderlich sein. ${ }^{78} \mathrm{Im}$ innerstaatlichen Verfahren muss die EMRK-Verletzung zumindest der Sache nach geltend gemacht werden (inhaltliche Rechtswegerschöpfung). ${ }^{79}$ Es ist nicht nötig, dass im innerstaatlichen Verfahren bereits die EMRK-Vorschrift genannt wurde.

72 EGMR, 30.6.2005, Nr. 45036/98, Rn 155 (Bosphorus/Irland), NJW 2006, 197.

${ }^{73}$ Vgl. die Widerlegung der Vermutung im Fall EGMR, 12.9.2012, Nr. 10593/08, Rn 172 (Nada/Schweiz), NJOZ 2013, 1183.

${ }^{74}$ Vgl. IGH, 21.3.1959, Interhandel Case (Preliminary Objections), ICJ Rep. 1959, 6, 27.

${ }^{75}$ Zu Art. 13 vgl. Peters/Altwicker, EMRK, § 22.

${ }^{76}$ EGMR, 19.2.2009, Nr. 2334/03, Rn 40 (Kozacığlu/Türkei).

${ }^{77}$ Siehe z. B. EGMR, 29.6.1999, Nr. 47547/99 (Yazdi/Deutschland).

${ }^{78}$ EGMR, 10.8.2006, Nr. 75737/01, Rn 28 ff (Schwarzenberger/Deutschland), NJW 2007, 3553.

${ }^{79}$ EGMR, 1.6.2010, Nr. 22978/05, Rn 142 (Gäfgen/Deutschland), NJW 2010, 3145. 
Anne Peters/Tilmann Altwicker, in: Jörg Philipp Terhechte (Hrsg.), Enyzklopädie des Europarechts, Band 3 (Baden-Baden: Nomos 2014)

29

Die innerstaatlichen Rechtsbehelfe müssen im Einklang mit den nationalen Prozessvorschriften, insbesondere form- und fristgerecht, eingelegt worden sein. Wurden nationalrechtliche Prozessvoraussetzungen nicht eingehalten und deshalb das innerstaatliche Rechtsmittel von einem staatlichen Gericht als unzulässig abgewiesen, führt dies in der Regel dazu, dass der EGMR eine Rechtswegerschöpfung nach Art. 35 Abs. 1 EMRK verneint, und damit ist die Beschwerde vor dem EGMR unzulässig. ${ }^{80}$

\section{b) Zugänglichkeit und Wirksamkeit}

\section{0}

Die Rechtswegerschöpfungsvoraussetzung wird dadurch eingeschränkt bzw. abgemildert, dass die nationalen Rechtsbehelfe zugänglich und wirksam sein müssen. Unzugängliche oder unwirksame nationale Rechtsbehelfe muss der Beschwerdeführer nicht ergreifen, sondern er darf direkt Beschwerde beim EGMR einlegen. Die (faktische) Zugånglichkeit des Rechtsbehelfs ist problematisch, wenn einem mittellosen Beschwerdeführer keine Prozesskostenhilfe gewährt wird ${ }^{81}$ oder wenn dieser der Verfahrenssprache nicht mächtig ist und er keine Unterstützung durch einen Dolmetscher erhält. Neben Hindernissen, die in der Person des Beschwerdeführers begründet sind, können auch politische Umstände zur Unzugänglichkeit führen. ${ }^{82}$ Die Wirksamkeit ist zweifelhaft, wenn der Rechtsbehelf ungeeignet ist, die Aufhebung bzw.

Rechtswidrigkeitsfeststellung des angegriffenen Hoheitsakts zu bewirken. Es muss sich mit anderen Worten um eine echte Abhilfemöglichkeit handeln - wenn dies nicht der Fall ist, muss der Beschwerdeführer nicht versuchen, sie zu ergreifen. Der EGMR hält in neuerer Rechtsprechung die Verfassungsbeschwerde an das BVerfG bei Fragen der überlangen Verfahrensdauer in Zivil- und Strafprozessen in der Regel nicht für einen wirksamen Rechtsbehelf. ${ }^{83}$ Unwirksam ist auch ein Rechtsbehelf, der von vornherein keine Aussicht auf Erfolg hat. ${ }^{84}$ Einfache Zweifel an der Erfolgsaussicht reichen allerdings nicht aus. ${ }^{85}$ Schließlich handelt es sich auch um keinen wirksamen Rechtsbehelf, wenn der Staat sich völlig untätig verhält (z.B. keinerlei Ermittlungen anstellt). ${ }^{86}$ In allen diesen Situationen ist unmittelbar eine Beschwerde an den EGMR statthaft.

31

Die Beweislast ist im Kontext der Rechtswegerschöpfung wie folgt verteilt: Die gesamte Zulässigkeit einer Beschwerde (einschließlich der Rechtswegerschöpfungsvoraussetzung) wird vom EGMR von Amts wegen

${ }^{80}$ EGMR, 14.4.2009, Nr. 18297/08 (Borisenko u.a./Armenien). Vgl. aber EGMR, 23.10.1996, Nr.

17748/91, Rn 31 (Ankerl/Schweiz), ÖJZ 1997, 475.

${ }^{81}$ EGMR, 9.10.1979, Nr. 6289/73, Rn 24 (Airey/Irland), EuGRZ 1979, 626.

${ }^{82}$ EGMR, 16.9.1996, Nr. 21893/93, Rn 70-77 (Akdivar/Türkei).

${ }^{83} \mathrm{~S}$ die Zusammenfassung in EGMR, 13.11.2008, Nr. 26073/03, Rn 56 ff (Ommer/Deutschland Nr. 2).

${ }^{84}$ EGMR, 25.8.1987, Nr. 10282/83, Rn 32 (Englert/Deutschland), NJW 1988, 3257.

${ }^{85}$ EGMR, 28.9.1999, Nr. 29340/95, Rn 43 f (Civet/Frankreich), NJW 2001, 54; vgl. auch EGMR, 19.3.2002, Nr. 77631/01 (Milosevic/Niederlande), EuGRZ 2002, 131.

${ }^{86}$ EGMR, 28.7.1999, Nr. 25803/94, Rn 74-81 (Selmouni/Frankreich), NJW 2001, 56. 
Anne Peters/Tilmann Altwicker, in: Jörg Philipp Terhechte (Hrsg.), Enyzklopädie des Europarechts, Band 3 (Baden-Baden: Nomos 2014)

geprüft. Der Beschwerdeführer muss die von ihm ergriffenen Rechtsmittel aufzählen und belegen. Die Regierung kann dann als Zulässigkeitseinwand (,preliminary objection“) vorbringen, der Rechtsweg sei nicht erschöpft. Sie muss die weiteren nationalen Rechtsmittel nennen und nachweisen, dass diese auch zugänglich und wirksam gewesen wären (Beweislast beim Staat). Wenn die Regierung das getan hat, muss wiederum der Beschwerdeführer seinerseits zeigen, dass diese Rechtsmittel ihm im konkreten Fall nicht effektiv zur Verfügung standen oder dass ihn bestimmte Umstände von der Verpflichtung zur Erschöpfung befreiten. Wenn der Staat die Zugänglichkeit und Wirksamkeit des nationalen Rechtsbehelfs nicht beweisen kann, gilt der Rechtsweg als erschöpft. ${ }^{87}$

\section{Form (Regel 45 EGMR-VerfO) und Frist (Art. 35 Abs. 1 EMRK)}

\section{2}

Die Beschwerde muss schriftlich eingereicht und vom Beschwerdeführer oder seinem Vertreter unterzeichnet werden (Regel 45 EGMR-VerfO). Zur Wahrung der Frist des Art. 35 Abs. 1 EMRK muss das von der Kanzlei zur Verfügung gestellte Formular ${ }^{88}$ verwendet werden, in dem u.a. der Gegenstand der Beschwerde (Identität des Beschwerdeführers, Benennung des Beschwerdegegners, zusammenfassende Sachverhaltsdarstellung sowie Ausführungen zur Beschwer) dargelegt wird (Regel 47 Abs. 1 S. 2 u. Regel 47 Abs. 6 EGMR-VerfO). ${ }^{89}$ Die Individualbeschwerde darf nur innerhalb einer Frist von sechs Monaten nach der endgültigen innerstaatlichen Entscheidung eingelegt werden (Art. 35 Abs. 1 EMRK). ${ }^{90}$ Das Fristerfordernis dient der Rechtssicherheit und wird vom Gerichtshof von Amts wegen geprüft. ${ }^{91}$ Die Frist läuft in der Regel ab Zustellung der letztinstanzlichen innerstaatlichen Entscheidung (Entscheidungsgründe). ${ }^{92}$ Fristwahrend ist schon das Abschicken der Beschwerde (Poststempel), nicht erst der Zugang beim EGMR. ${ }^{93}$ Das Beschwerdformular kann auch per Fax eingereicht werden. ${ }^{94}$ Für die Fristberechnung gilt Folgendes: Die Frist beginnt mit dem auf die förmliche Bekanntgabe der Entscheidung (i.d.R. die Zustellung) folgenden Tag. Sie läuft in sechs Kalendermonaten ab, unabhängig davon, ob das Fristende auf einen Wochenend- oder Feiertag fällt. ${ }^{95}$

${ }^{87}$ EGMR, 15.1.2009, Nr. 46598/06, Rn 35 (Tomašic u.a./Kroatien).

${ }^{88}$ Das Beschwerdeformular (Regel 47 Abs. 1 S. 1 EGMR-VerfO) kann auf der Internetseite des EGMR (www.echr.coe.int) abgerufen werden.

${ }^{89}$ Vor dem 1.1.2014 genügte zunächst ein formloses Schreiben, s. Regel 47Abs. 5 S. 1 EGMR-VerfO a.F.

${ }^{90}$ Das noch nicht in Kraft getretene 15. Protokoll (Fn. 57) reduziert die Beschwerdefrist auf vier Monate. Dies kann in Fällen, die eine komplexe EMRK-Dogmatik aufweisen (z.B. Art. 6 EMRK), bedenklich kurz sein.

${ }^{91}$ EGMR, 10.11.2009, Nr. 21425/06 (Otto/Deutschland).

92 EGMR, 29.8.1997, Nr. 22714/93, Rn 33 (Worm/Österreich), MR 1997, 295.

${ }^{93}$ EGMR, 11.5.2006, Nr. 31753/02 (Kaya/Deutschland).

${ }^{94}$ S., mutatis mutandis, EGMR, 1.6.2010, Nr. 65938/09, Rn 21 ff (Kemevuako/Niederlande).

${ }^{95}$ EGMR, 10.11.2009, Nr. 21425/06 (Otto/Deutschland). 
Anne Peters/Tilmann Altwicker, in: Jörg Philipp Terhechte (Hrsg.), Enyzklopädie des Europarechts, Band 3 (Baden-Baden: Nomos 2014)

\title{
9. Negativvoraussetzungen der Zulässigkeit
}

\author{
a) Anonyme Beschwerde (Art. 35 Abs. 2 lit. a) EMRK)
}

33

Der Gerichtshof weist anonyme Beschwerden, d.h. solche, aus denen der Beschwerdeführer nicht hervorgeht, als unzulässig zurück. ${ }^{96}$ Der Gerichtshof kann entscheiden, dass die Beschwerde anonymisiert wird, z.B. bei befürchteten Sanktionen gegen den Beschwerdeführer (Regel 47 Abs. 4 S. 2 EGMR-VerfO).

\section{b) Res iudicata und Litispendenz (Art. 35 Abs. 2 lit. b) EMRK)}

\section{4}

Eine Beschwerde, die im Wesentlichen mit einer schon vorher vom Gerichtshof geprüften Beschwerde übereinstimmt (res iudicata), wird als unzulässig zurückgewiesen. Eine wesentliche Übereinstimmung liegt vor, wenn die Parteien, die Beschwer sowie der Sachverhalt identisch sind. ${ }^{97}$ Unter gewissen Voraussetzungen kann die Wiederaufnahme des Beschwerdeverfahrens gemäß Regel 80 Abs. 1 EGMR-VerfO beantragt werden, wenn eine Tatsache erst später bekannt wird, die geeignet gewesen wäre, einen maßgeblichen Einfluss auf den Ausgang einer bereits entschiedenen Rechtssache auszuüben. Ebenso ist eine Beschwerde unzulässig, wenn diese der Sache nach schon einer anderen internationalen Untersuchungs- oder Vergleichsinstanz unterbreitet worden ist und keine neuen Tatsachen enthält (Litispendenz). ${ }^{98}$ Solche anderen Instanzen sind beispielsweise der UN-Menschenrechtsausschuss (Art. 28 IPBürg) ${ }^{99}$ oder die ILO-

Streitbeilegungsgremien, ${ }^{100}$ nicht aber ein Verfahren vor dem Europäischen Ausschuss zur Verhütung von Folter und unmenschlicher Behandlung (da dies kein justizförmiges Verfahren ist). ${ }^{101}$

c) Offensichtliche Unbegründetheit der Beschwerde (Art. 35 Abs. 3 lit. a) Alt. 2 EMRK)

\section{5}

Der Gerichtshof weist die Beschwerde auch dann als unzulässig zurück, wenn sie sich im Vorgriff auf die materielle Prüfung als offensichtlich unbegründet erweist. Der Gerichtshof macht viel Gebrauch von diesem Unzulässigkeitsgrund. Die „offensichtliche“ Unbegründetheit setzt nicht voraus, dass die Ablehnung einer Konventionsverletzung evident sein muss. In der Praxis lassen sich vier Situationen der offensichtlichen

${ }^{96}$ EGMR, 15.9.2009, Nr. 7245/09 („Blondje“/ Niederlande).

${ }^{97}$ EGMR, 30.6.2009, Nr. 32772/02, Rn 63 (Verein gegen Tierfabriken Schweiz (VgT)/Schweiz (Nr. 2)), NJW 2010, 3699.

${ }^{98}$ Gute Zusammenfassung der Rspr. in EGMR, 21.5.2013, Nr. 59253/11, Rn 26 ff (Poa u.a./Vereinigtes Königreich).

${ }^{99}$ EKMR, 6.7.1992, Nr. 17512/90 (Calcerrada Fornieles et Caheza Mato/Spanien).

${ }^{100}$ EKMR, 12.10.1992, Nr. 16358/90 (Cereceda Martin u.a./Spanien).

${ }^{101}$ EGMR, 3.6.2008, Nr. 24408/03 (Zagaria/Italien). 
Anne Peters/Tilmann Altwicker, in: Jörg Philipp Terhechte (Hrsg.), Enyzklopädie des Europarechts, Band 3 (Baden-Baden: Nomos 2014)

Unbegründetheit unterscheiden: ${ }^{102}$ Erstens nicht hinreichend substantiierte Beschwerden (vor allem, wenn die Beschwerdeschrift nicht auf die inhaltlichen Minimalerfordernisse gem. Regel 47 Abs. 1 S. 2 EGMRVerfO eingeht), ${ }^{103}$ zweitens sog. „Vierte-Instanz“-Fälle, d.h. Beschwerden, in denen (ausschließlich) die falsche Anwendung des innerstaatlichen Rechts bzw. die fehlerhafte Sachaufklärung durch nationale Gerichte gerügt wird, ${ }^{104}$ drittens das offensichtliche Fehlen einer Konventionsverletzung (z.B. bei offensichtlich gerechtfertigten Hoheitsakten; bei Vorliegen einer gefestigten, die Konventionsverletzung in ähnlichen Fällen ablehnenden Rechtsprechung des EGMR) ${ }^{105}$ und viertens der offensichtlich unzutreffende bzw. unwahrscheinliche Sachverhaltsvortrag bzw. der nicht beweisbare Sachverhalt. ${ }^{106}$

d) Missbrauch des Beschwerderechts (Art. 35 Abs. 3 lit. a) Alt. 3 EMRK)

36

In seltenen Fällen hat der Gerichtshof die Beschwerde für unzulässig erklärt, wenn im Prozessverhalten des Beschwerdeführers ein Missbrauch des Beschwerderechts zu sehen ist. ${ }^{107}$ Ein Missbrauch liegt vor, wenn der Beschwerdeführer sich wissentlich auf falsche Tatsachen stützt oder gefälschte Dokumente vorlegt. ${ }^{108}$ Ferner handelt es sich um einen Missbrauch des Beschwerderechts, wenn im Verhalten des Beschwerdeführers eine Missachtung des Gerichtshofs zum Ausdruck kommt. ${ }^{109}$

\section{Staatenbeschwerde}

\section{7}

Die Staatenbeschwerde ermöglicht es allen Vertragsstaaten, den EGMR wegen behaupteter Verletzungen der Konvention oder ihrer Protokolle durch eine andere Vertragspartei anzurufen (Art. 33 EMRK). ${ }^{110}$ Der Anteil der Staatenbeschwerden ist äußerst gering. Bisher sind insgesamt nur gut 20 Staatenbeschwerden eingelegt worden. Ein Vertragsstaat kann die Staatenbeschwerde zum einen als Instrument des diplomatischen Schutzes verwenden, indem er die Konventionsverletzung eigener Staatsangehöriger durch eine andere Vertragspartei rügt. ${ }^{111}$ Zum anderen gibt die Staatenbeschwerde den Vertragsstaaten die Möglichkeit, eine „Wächterfunktion“ wahrzunehmen: Jeder Vertragsstaat kann, ohne dass eigene Interessen oder Rechte beeinträchtigt sein müssten, die Verletzung der EMRK oder ihrer Protokolle als europäischem ordre public

102 Siehe EGMR, Leitfaden zu den Zulässigkeitsvoraussetzungen, Straßburg 2010, S. 68 ff.

${ }^{103}$ EGMR, 1.7.2010, Nr. 17674/02 u.a., Rn 152 (Davydov u.a./Ukraine).

${ }^{104}$ EGMR, 28.1.2011, Nr. 18160/06, Rn 2 (Miklošević/Serbien).

${ }^{105}$ EGMR, 28.10.2010, Nr. 4241/03, Rn 54 (Trofimchuk/Ukraine).

${ }^{106}$ EGMR, 23.3.2010, Nr. 28439/03, Rn 35 ff (Duman/Türkei).

107 S. EGMR, 15.9.2009, Nr. 798/05, Rn 62 ff (Miroḷubovs u.a./Litauen), NVwZ 2010, 1541.

${ }^{108}$ EGMR, 5.10.2000, Nr. 31365/96, Rn 36 (Varbano/Bulgarien).

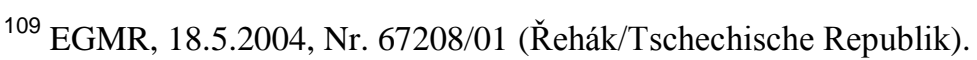

${ }^{110} \mathrm{Zu}$ den Zulässigkeitsvoraussetzungen s. Peters/Altwicker, EMRK, § 36 Rn 3.

${ }^{111}$ EGMR, 5.4.2000, Nr. 34382/97 (Dänemark/Türkei), EuGRZ 2000, 619 (gütliche Einigung). 
Anne Peters/Tilmann Altwicker, in: Jörg Philipp Terhechte (Hrsg.), Enyzklopädie des Europarechts, Band 3 (Baden-Baden: Nomos 2014)

rügen (siehe den Wortlaut des Art. 33 EMRK: „Jeder“). ${ }^{112}$ Die EMRK fungiert also als objektive Ordnung. Beispielsweise erhoben im Jahr 1967 Dänemark, Norwegen, Schweden und die Niederlande Beschwerde vor der damaligen Kommission gegen das griechische Obristenregime. ${ }^{113}$ Insbesondere Zypern hat mehrfach gegen die Türkei im Zusammenhang mit der Besetzung Nordzyperns geklagt. ${ }^{114}$ Die Zulässigkeitsvoraussetzungen der Staatenbeschwerde sind in Art. 33 und 35 Abs. 1 EMRK geregelt. Art. 35 Abs. 3 EMRK ist nicht anwendbar. ${ }^{115}$

\section{Der Verfahrensgang}

\section{8}

Der Verfahrensgang bei der Individualbeschwerde und der viel selteneren Staatenbeschwerde ist im Wesentlichen ähnlich. ${ }^{116}$ Die folgenden Ausführungen beziehen sich primär auf die Individualbeschwerde. Der erste Schritt ist das erste Schreiben des Beschwerdeführers an die Kanzlei des EGMR. Die Kanzlei prüft dann, ob die Beschwerde offensichtlich unzulässig oder offensichtlich unbegründet ist oder bereits entschieden wurde (vgl. Art. 35 Abs. 2 lit. b) EMRK). Die Kanzlei gibt der Beschwerde ein Aktenzeichen und registriert diese. Der Präsident des Gerichtshofs weist die Beschwerde einer Sektion zu (Regel 52 Abs. 1 EGMR-VerfO).

39

Der Einzelrichter kommt zum Zuge, falls der Berichterstatter die Beschwerde für unzulässig hielt. Der Einzelrichter erklärt die Beschwerde endgültig für unzulässig oder streicht sie im Register, wenn diese Entscheidung ohne weitere Prüfung ergehen kann (Art. 27 Abs. 1 EMRK; Regel 52a Abs. 1 EGMR-VerfO).

40

Der Ausschuss mit drei Richtern kann eine Individualbeschwerde einstimmig für endgültig unzulässig erklären oder im Register streichen (Art. 28 Abs. 1 lit. a) EMRK; Regel 53 Abs. 1 EGMR-VerfO). Der Ausschuss kann die Beschwerde allerdings auch für zulässig erklären und zugleich ein Urteil über die Begründetheit fällen, wenn zu der Sache eine gefestigte Rechtsprechung des Gerichtshofs besteht (Art. 28 Abs. 1 lit. b) EMRK; Regel 53 Abs. 2 EGMR-VerfO).

Sofern weder Einzelrichter noch Dreierausschuss endgültig über die Sache entscheiden, kommt die Individualbeschwerde vor eine Kammer mit sieben Richtern (Art. 29 Abs. 1 EMRK). Sofern die Kammer die Beschwerde für zulässig hält und eine weitere Sachaufklärung nicht notwendig erscheint, stellt sie die Beschwerde dem Vertragsstaat zu, gegen den sie sich richtet (Regel 54 Abs. 2 lit. b) EGMR-VerfO). In diesem

\footnotetext{
${ }^{112}$ Bsp.: EGMR, 24.1.1968, Nr. 3321/67 u.a. (Dänemark, Norwegen, Schweden u. Niederlande/Griechenland). Vgl. E. Klein, in: Merten/Papier (Hg.), § 150, Rn 75 f.

${ }^{113}$ EGMR, 24.1.1968, Nr. 3321/67 u.a. (Dänemark, Norwegen, Schweden und Niederlande/Griechenland).

${ }^{114}$ Zuletzt EGMR, 10.5.2001, Nr. 25781/94 (Zypern/Türkei).

${ }^{115}$ EGMR, 13.12.2011, Nr. 38263/08, Rn 64 (Georgien/Russland Nr. 2).

${ }^{116}$ Die Verfahrensordnung des EGMR enthält gemeinsame und spezielle Vorschriften für beide Verfahrensarten.
} 
Anne Peters/Tilmann Altwicker, in: Jörg Philipp Terhechte (Hrsg.), Enyzklopädie des Europarechts, Band 3 (Baden-Baden: Nomos 2014)

Fall wird der betreffende Vertragsstaat zu einer Stellungnahme aufgefordert. In dieser Stellungnahme oder spätestens in einer mündlichen Verhandlung muss der Vertragsstaat alle eventuellen Einwände gegen die Zulässigkeit vorbringen, da er sonst mit diesen Einwänden präkludiert ist (Regel 55 EGMR-VerfO). Ab dem Zeitpunkt der Zustellung muss der Beschwerdeführer anwaltlich vertreten sein, sofern nicht der Kammerpräsident anders entscheidet (Regel 36 Abs. 2 EGMR-VerfO). Die Kammer prüft die Zulässigkeit und die Begründetheit zusammen.

41

Parallel dazu versucht der Gerichtshof, auf eine güitliche Einigung der Parteien hinzuwirken (Art. 39 EMRK; Regel 62 EGMR-VerfO); dies gilt für Individual- und Staatenbeschwerden. Das Verfahren ist vertraulich (Art. 39 Abs. 2 EMRK). Kommt es zu einer gütlichen Einigung, so streicht der Gerichtshof die Beschwerde im Register, nachdem er sich davon überzeugt hat, dass die Einigung auf der Grundlage der Achtung der Menschenrechte zustande gekommen ist (Art. 39 Abs. 1 EMRK; Regel 62 Abs. 3 EGMR-VerfO). Die Streichung einer Beschwerde aufgrund einer gütlichen Einigung erfolgt durch eine Entscheidung, deren Durchführung vom Ministerkomitee überwacht wird (Art. 39 Abs. 3 u. 4 EMRK; Regel 43 Abs. 3 EGMRVerfO). In dieser Entscheidung des Gerichtshofs wird keine Konventionsverletzung festgestellt, sondern es wird eine individuelle, rechtlich verbindliche Vereinbarung zwischen den Parteien getroffen (in der Regel über die Zahlung einer Entschädigung).

\section{2}

Kommt es nicht zu einer gütlichen Einigung, wird die Beschwerde weiter geprüft. Das Verfahren ist in aller Regel ein Aktenprozess; nur sehr selten findet eine mündliche Verhandlung statt. ${ }^{117}$ Falls erforderlich, kann der Gerichtshof Ermittlungen vor Ort vornehmen, um sich z.B. ein Bild von den Haftbedingungen in einem Gefängnis zu machen (Art. 38 EMRK). In jedem Verfahrensstadium kann der Gerichtshof eine zuvor für zulässig erklärte Beschwerde für unzulässig erklären (Art. 35 Abs. 4 S. 2 EMRK). Dann ergeht jedoch keine Zulässigkeitsentscheidung mehr, sondern ein Urteil. Der EGMR kann auch jederzeit Beschwerden aus bestimmten anderen Gründen streichen, z.B. wenn die Streitigkeit anderweit gelöst wurde (Art. 37 EMRK). ${ }^{118}$

43

Die Kammer kann die Beschwerde an die Große Kammer mit 17 Richtern abgeben, wenn sich eine schwerwiegende Auslegungsfrage stellt oder die Möglichkeit der Abweichung von früherer Rechtsprechung besteht, Art. 30 EMRK. Eine weitere bedeutsame Zuständigkeit der Großen Kammer wird durch Verweisung begründet, Art. 43 EMRK. Hier fungiert die Große Kammer als Kontrollinstanz nach einem Kammerurteil. Die Verweisung ist bei einer schwerwiegenden Auslegungsfrage oder einer Frage von allgemeiner Bedeutung möglich. Die Rechtssache muss erst durch einen Vorprüfungsausschuss von fünf Richtern der Großen Kammer angenommen werden, Art. 43 Abs. 2 EMRK; Regel 24 Abs. 5 EGMR-VerfO. Weil die Richter der Großen Kammer teilidentisch mit denen der vorher urteilenden Kammer sind (s. Art. 26 Abs. 5

${ }^{117}$ Vgl. für die Individualbeschwerde Regel 54 Abs. 5 u. 59 EGMR-VerfO; für die Staatenbeschwerde Regel 51 Abs. 1 und 5 sowie Regel 58 Abs. 2 EGMR-VerfO.

${ }^{118}$ Z.B. EGMR, 30.11.2010, Nr. 36397/07 (Görgülü u. Fischer/Deutschland), EuGRZ 2011, 264. 
Anne Peters/Tilmann Altwicker, in: Jörg Philipp Terhechte (Hrsg.), Enyzklopädie des Europarechts, Band 3 (Baden-Baden: Nomos 2014)

S. 2 EMRK) und ein Annahmeermessen (s. Art. 43 Abs. 2 EMRK) besteht, handelt es sich bei der Kontrolle nicht um ein echtes Rechtsmittel. ${ }^{119}$

44

Mittlerweile ist die Beteiligung Dritter am Verfahren üblich. Diese ist bei beiden Beschwerdearten möglich, wenn dies ,im Interesse der Rechtspflege“ liegt (Art. 36 Abs. 2 EMRK; Regel 44 Abs. 3 EGMR-VerfO). ${ }^{120}$ Drittbeteiligte können entweder andere Vertragsstaaten, NGOs, ${ }^{121}$ der Menschenrechtskommissar des Europarats, ${ }^{122}$ internationale Organisationen oder sogar andere Privatpersonen sein.

\section{Gutachtenverfahren}

\section{5}

Auf Antrag des Ministerkomitees erstattet die Große Kammer ein Gutachten über Rechtsfragen, welche die Auslegung der EMRK und der Protokolle betreffen (Art. 47 ff. EMRK, Regel 82 ff. EGMR-VerfO). Bislang hat der Gerichtshof nur zwei Gutachten erstattet. ${ }^{123}$ Die seltene Anwendung hängt mit den Voraussetzungen in Art. 47 Abs. 2 EMRK zusammen: Das Gutachten darf erstens keine Fragen zum Gegenstand haben, die sich auf den Inhalt oder das Ausmaß der Konventionsrechte beziehen (inhaltliche Beschränkung). Zweitens dürfen auch keine Fragen behandelt werden, über die der Gerichtshof oder das Ministerkomitee aufgrund eines nach der Konvention eingeleiteten Verfahrens zu entscheiden haben könnte (verfahrensrechtliche Beschränkung). Das Gutachtenverfahren ist also subsidiär zu den streitigen Beschwerdeverfahren und auch zum Urteilsbefolgungsverfahren nach Art. 46 Abs. 4 und Abs. 5 EMRK. In der Praxis hat dies dazu geführt, dass Gutachtenanfragen allein verfahrensrechtliche Probleme zum Gegenstand hatten: Beide Gutachten betrafen die Wahl der Richter zum EGMR (vgl. Art. 21 f. EMRK). ${ }^{124}$ Das noch nicht in Kraft befindliche 16. Protokoll sieht nunmehr eine erhebliche Ausweitung des Gutachtenverfahrens vor. ${ }^{125}$ Um den Dialog der obersten Gerichte in Konventionsrechtsfragen zu verbessern, sollen die obersten Gerichte der

${ }^{119}$ Die Große Kammer ist ferner für das neue Verfahren wegen Nichtbefolgung eines Urteils zuständig, Art. 31 lit. b) i.V.m. Art. 46 Abs. 4, 5 EMRK. Schließlich ist die Große Kammer zuständig für Anträge auf Erstattung von Gutachten, Art. 47 EMRK, s. unten.

${ }^{120}$ Die Aufforderung zur Stellungnahme kann von Amts wegen ergehen oder auf Antrag einer der Streitparteien (Regel 44 Abs. 3 a) und b) EGMR-VerfO).

${ }^{121}$ In schwierigen Fällen sind z.T. auch mehr als eine NGO beteiligt worden. So z.B. im Fall EGMR, 6.7.2005, Nr. 43577/98 u.a., Rn 8 (Nachova u.a./Bulgarien), EuGRZ 2005, 693.

${ }^{122}$ Erstmals beteiligt an EGMR, 21.1.2011, Nr. 30696/09 (M.S.S./Belgien u. Griechenland).

${ }^{123}$ In einem Fall lehnte der EGMR die Erstattung eines Gutachtens ab (2.6.2004), NJW 2005, 123.

${ }^{124}$ Im ersten Gutachten (EGMR, 12.2.2008 = NJW 2009, 2109) ging es im Wesentlichen um die Frage, ob die Parlamentarische Versammlung eine Kandidatenliste für die Richterwahl aus Gründen der Gleichstellung der Geschlechter zurückweisen könne. Das zweite Gutachten (EGMR, 22.1.2010) betraf Fragen im Zusammenhang mit der Zurückziehung einer Kandidatenliste durch eine Vertragspartei.

125 16. Protokoll zur EMRK, 2.10.2013. Zu den Entwicklungen s. das „Reflection Paper“ des EGMR, http://www.echr.coe.int/Documents/2013_Courts_advisory_jurisdiction_ENG.pdf (6.9.2013). 
Anne Peters/Tilmann Altwicker, in: Jörg Philipp Terhechte (Hrsg.), Enyzklopädie des Europarechts, Band 3 (Baden-Baden: Nomos 2014)

Vertragsstaaten ein nicht verbindliches Gutachten des EGMR anfordern können. Es wird von der Großen Kammer nach einem Vorprüfungsverfahren, in welchem die Anfrage mit einer Begründung zurückgewiesen werden kann, erstattet. Gutachten können nur im Zusammenhang mit einem laufenden innerstaatlichen Verfahren angefordert werden; eine abstrakte Normenkontrolle durch den EGMR ist weiterhin ausgeschlossen. Die Erweiterung des Gutachtenverfahrens ist zu begrüßen. ${ }^{126}$

\section{Vorläufiger Rechtsschutz}

46

Einstweilige Anordnungen sind nach Regel 39 EGMR-VerfO möglich. Diese erlässt die zuständige Kammer, der Sektionspräsident oder ein besonders ernannter Richter (duty judge) auf Antrag einer Partei, einer anderen betroffenen Person oder auch von Amts wegen. Eine einstweilige Anordnung dient dazu, eine Präjudizierung der Hauptsache - und damit eine Vereitelung des Beschwerderechts - durch die Schaffung vollendeter Tatsachen zu verhindern. Der betreffende Staat wird verpflichtet, jegliche Handlungen zu unterlassen, die geeignet sind, die Wirksamkeit des Urteils in der Hauptsache zu beeinträchtigen. ${ }^{127}$ Der Gerichtshof wendet Regel 39 EGMR-VerfO zunehmend stark restriktiv an. ${ }^{128}$ Voraussetzung ist die unmittelbare Gefahr des Eintritts eines irreparablen Schadens. ${ }^{129}$ Solche Anordnungen erfolgen typischerweise in Ausweisungsfällen, wenn dem Betroffenen im Zielstaat körperliche Misshandlungen oder sogar die Todesstrafe drohen. ${ }^{130}$ Einstweilige Anordnungen nach Regel 39 EGMR-VerfO sind rechtsverbindlich. ${ }^{131}$ Befolgt der betreffende Vertragsstaat eine einstweilige Anordnung des EGMR nicht, so kann der Gerichtshof eine Verletzung des Rechts aus Art. 34 EMRK (Recht auf Individualbeschwerde) feststellen. ${ }^{132}$ In engen Ausnahmefällen führt die Nichtbefolgung einer einstweiligen Anordnung jedoch nicht zu einer Verletzung von Art. 34 EMRK: ${ }^{133}$ Es muss erstens ein objektiver Hinderungsgrund bestanden haben, der die Befolgung unmöglich machte. Zweitens muss der Vertragsstaat alle vernünftigen Schritte unternommen haben, um den Hinderungsgrund der Umsetzung der Anordnung zu beseitigen. Drittens muss der Vertragsstaat den Gerichtshof über die getroffenen Maßnahmen zur Befolgung der einstweiligen Anordnung laufend unterrichten.

\footnotetext{
${ }^{126}$ Näher Peters/Altwicker, EMRK, § 36 Rn 7.

${ }^{127}$ EGMR, 4.2.2005, Nr. 46827/99 u.a., Rn 128 (Mamatkulov u. Askarov/Türkei), EuGRZ 2005, 357. .

${ }^{128}$ Im Jahr 2012 lagen 1.972 Anträge vor, ein deutlicher Rückgang zu den Vorjahren. Davon wurden nur 103 (ca. $5 \%$ ) positiv beschieden.

${ }^{129}$ EGMR, 2.3.2010, Nr. 61498/08, Rn 160 (Al-Saadoon und Mufdhi/Vereinigtes Königreich).

${ }^{130}$ Z.B. EGMR, 24.2.2009, Nr. 246/07, Rn 69 ff (Ben Khemais/Italien).

${ }^{131}$ EGMR, 4.2.2005, Nr. $46827 / 99$ u.a., Rn 109-128 (Mamatkulov u. Askarov/Türkei).

132 EGMR, 22.12.2008, Nr. 46468/06, Rn 228 ff (Aleksanyan/Russland).

${ }^{133}$ EGMR, 2.3.2010, Nr. 61498/08, Rn 161 (Al-Saadoon und Mufdhi/Vereinigtes Königreich).
} 
Anne Peters/Tilmann Altwicker, in: Jörg Philipp Terhechte (Hrsg.), Enyzklopädie des Europarechts, Band 3 (Baden-Baden: Nomos 2014)

\section{Urteilsinhalt und Urteilswirkungen}

\section{Urteilsinhalte}

\section{a) Feststellung der Konventionsverletzung}

\section{7}

EGMR-Urteile sind im Kern Feststellungsurteile (vgl. den Wortlaut des Art. 41 EMRK: „Stellt der Gerichtshof fest ...“). Der Urteilstenor lautet also z.B.: „Aus diesen Gründen stellt der Gerichtshof fest, dass eine/keine Verletzung des Artikels 10 vorliegt.“ Die Urteile des EGMR haben weder eine kassatorische (aufhebende) noch eine gestaltende Wirkung. Der Gerichtshof kann daher keine innerstaatlichen Gesetze, Urteile oder Verwaltungsakte aufheben.

\section{b) Gerechte Entschädigung, Art. 41 EMRK}

48

Daneben kann der EGMR im Urteil zusätzlich nach Art. 41 EMRK der verletzten Partei eine gerechte Geldentschädigung zusprechen. Diese muss der ,verurteilte“ Staat zahlen. Bei Säumigkeit oder Weigerung des Staates muss die Entschädigung vom Opfer gegebenenfalls innerstaatlich eingeklagt werden. In Deutschland ist hierfür der ordentliche Rechtsweg nach $§ 40$ Abs. 2 S. 1 Var. 3 VwGO gegeben (Schadenersatz wegen Verletzung nichtvertraglicher öffentlich-rechtlicher Pflichten). ${ }^{134}$ Die Entschädigung umfasst zunächst den materiellen und immateriellen Schadenersatz. Die Höhe der Entschädigung nach Art. 41 EMRK richtet sich nach der konkreten Rechtsverletzung. Der Gerichtshof entscheidet darüber nach Billigkeitsgesichtspunkten. $^{135}$

49

Mit Art. 41 EMRK kann der Beschwerdeführer zudem Ersatz der Kosten und Auslagen verlangen. Das Verfahren vor dem EGMR selbst ist gerichtsgebührenfrei. Die Kosten des Gerichtshofs tragen der Europarat und somit die Vertragsstaaten (Art. 50 EMRK). Als Kosten und Auslagen kann der Beschwerdeführer v.a. die Kosten der Rechtsverfolgung vor nationalen Instanzen (Gerichtskosten, Kosten für Sachverständige) und Rechtsanwaltskosten geltend machen. Voraussetzungen sind, dass (1) die Kosten tatsächlich angefallen sind (dieses ist z.B. nicht gegeben, wenn die Kosten von einer Rechtsschutzversicherung getragen wurden), (2) im Zusammenhang mit der Konventionsverletzung stehen sowie (3) angemessen sind. ${ }^{136}$

\footnotetext{
${ }^{134}$ Siehe z.B. BGH, 24.3.2011, IX ZR 180/10, Rn 22 u. 27.

${ }^{135}$ Für einen Überblick über die für immaterielle Schäden gewährten Entschädigungssummen vgl. die Aufstellung bei Karpenstein/Mayer/Wenzel, EMRK, Art. 41, Rn 27 ff.

${ }^{136}$ Vgl. Karpenstein/Mayer/Wenzel, EMRK, Art. 41, Rn 48 ff.
} 
Anne Peters/Tilmann Altwicker, in: Jörg Philipp Terhechte (Hrsg.), Enyzklopädie des Europarechts, Band 3 (Baden-Baden: Nomos 2014)

\section{c) Erweiterungen: Anordnung von Einzelmaßnahmen und Piloturteile}

50

In Einzelfällen ordnet der EGMR im Tenor konkrete Umsetzungsmaßnahmen an, z.B. die Freilassung einer widerrechtlich inhaftierten Person. ${ }^{137}$ Es ist umstritten, ob der Gerichtshof zur Anordnung von Einzelmaßnahmen im Urteil überhaupt befugt ist. Dafür sprechen folgende Gründe: Erstens beabsichtigten die Vertragsstaaten mit der Einführung des Urteilsbefolgungsverfahrens vor dem EGMR (Art. 46 Abs. 4 u. 5 EMRK) im Jahr 2010 eine stärkere Verrechtlichung des Urteilsbefolgungsprozesses. Zweitens sind nach allgemeinem Völkerrecht (Recht der Staatenverantwortung) die Konventionsstaaten verpflichtet, einen konventionsgemäßen Zustand wiederherzustellen. Da dem Gerichtshof die primäre Aufgabe der Sicherstellung der Einhaltung der Konventionsverpflichtungen obliegt (Art. 19 EMRK) und seine durchsetzungsbezogenen Anordnungen wesentlich zur Wiederherstellung des konventionsgemäßen Zustands beitragen können, sollte der EGMR in Einzelfällen befugt sein, solche Maßnahmen anzuordnen. Drittens gilt der allgemeine Grundsatz, dass Konventionsrechte ,practical and effective“ sein müssen, auch in prozessualer Hinsicht. Das heißt, gerade die konventionsrechtlich vorgesehenen Durchsetzungsmechanismen sollen effektiv sein. Als Rechtsgrundlage zur Anordnung konkreter Maßnahmen im Einzelfall kommt eine Annexkompetenz in Betracht, die sich aus einer Gesamtschau der Art. 46, 41 und 19 EMRK ergibt. ${ }^{138}$

51

Im Fall Broniowski v. Polen (2004) hat der EGMR richterrechtlich, unter Berufung auf Art. 46 EMRK als Kompetenzgrundlage, das sog. Piloturteilsverfahren eingeführt. ${ }^{139}$ Einzelheiten sind seit 2011 in Regel 61 EGMR-VerfO geregelt. In diesen Fällen hat die Verletzung ihren Ursprung in einem „strukturellen oder systemischen Missstand“ in der Rechtsordnung des jeweiligen Vertragsstaates, der in der Gesetzeslage oder Verwaltungspraxis wurzelt. Die bisherigen Piloturteile betrafen unter anderem konventionswidrige Enteignungen einer großen Zahl von Einwohnern einer Region, ${ }^{140}$ das Mietrecht, ${ }^{141}$ unmenschliche und erniedrigende Haftbedingungen ${ }^{142}$ sowie fehlende Rechtsbehelfe gegen überlange Verfahrensdauer ${ }^{143}$ oder gegen Haftbedingungen. ${ }^{144}$ Bei Piloturteilsverfahren wird eine Individualbeschwerde ausgewählt, die stellvertretend für eine große Anzahl an gleich gelagerten Konventionsverletzungen steht. Müsste der Gerichtshof alle diese Fälle einzeln aburteilen, wäre er massiv überlastet. Ziel des Piloturteilsverfahrens ist also, den Staat

${ }^{137}$ EGMR, 8.4.2004, Nr. 71503/01 (Assanidze/Georgien), NJW 2005, 2207 (der Gerichtshof entschied „unanimously that the respondent State must secure the applicant's release at the earliest possible date").

${ }^{138}$ So auch Karpenstein/Mayer/Breuer, EMRK, 2011, Art. 46 Rn 8.

${ }^{139}$ EGMR, 22.4.2004, Nr. 31443/96, Rn 188 ff (Broniowski/Polen), NJW 2005, 2521. Vgl. Haider; Schmahl.

${ }^{140}$ EGMR, 22.4.2004, Nr. 31443/96 (Broniowski/Polen).

${ }^{141}$ EGMR, 19.6. 2006, Nr. 35014/97 (Hutten-Czapska/Polen).

${ }^{142}$ Gegen Art. 3 verstoßende Haftbedingungen: EGMR, 22.10.2009, Nr. 17885/04 (Orchowski/Polen).

${ }^{143}$ EGMR, 2.9.2010, Nr. 46344/06, Rn 59 ff (Rumpf/Deutschland), NJW 2010, 3355.

${ }^{144}$ EGMR, 10.1.212, Nr. 42525/07 u.a. (Ananyev u.a./Russland). 
Anne Peters/Tilmann Altwicker, in: Jörg Philipp Terhechte (Hrsg.), Enyzklopädie des Europarechts, Band 3 (Baden-Baden: Nomos 2014)

dazu zu bringen, das Strukturproblem zu beseitigen, die Fälle auf nationaler Ebene zu lösen und weitere Beschwerden an den Gerichtshof zu verhindern. ${ }^{145}$

52

Ein Piloturteil enthält im Tenor in der Regel drei Aussprüche: Erstens wird die Konventionsverletzung im Einzelfall festgestellt. Zweitens stellt der EGMR fest, dass diese auf einem systemischen Problem der Rechtsordnung des Vertragsstaates beruht. Meistens verpflichtet der Gerichtshof, drittens, den Vertragsstaat dazu, allgemeine Maßnahmen zur Beseitigung des systemischen Defekts zu ergreifen, und oft setzt er hierfür eine bestimmte Frist. ${ }^{146}$ So wurde Deutschland im Piloturteil Rumpf verpflichtet, innerhalb eines Jahres eine ,wirksame innerstaatliche Abhilfe“ für überlange Gerichtsverfahren zu schaffen. ${ }^{147}$ Daraufhin kann der EGMR eventuelle bereits anhängige parallele Beschwerdeverfahren einstweilig aussetzen (nach Regel 61 Abs. 6 EGMR-VerfO). Er setzt die Parallelverfahren jedoch nicht aus, wenn dies den Druck vom Staat zu nehmen droht und den Parallelbeschwerdeführern gegenüber unfair erscheint. ${ }^{148}$ Sobald im innerstaatlichen Recht der systemische Defekt so beseitigt wurde, dass die Abhilfe auch den Parallelbeschwerden zugutekommt (z.B. durch Anordnung einer Rückwirkung), werden diese Beschwerden ganz aus dem Register gestrichen.

\section{3}

Das Piloturteilsverfahren ist als Kompetenzüberschreitung des Gerichtshofs, als zu starke Anmaßung von quasi-verfassungsgerichtlichen Eingriffen in die nationalen Rechtssysteme kritisiert worden. Es besteht auch die Gefahr der Beschneidung des Rechtschutzes der Parallelbeschwerdeführer. Allerdings entspricht das Piloturteilsverfahren dem Subsidiaritätsprinzip, indem die Verantwortung für die Abstellung von Konventionsverletzungen klar dem betroffenen Staat zugewiesen wird. Dies funktioniert aber nur, wenn dieser kooperationswillig ist.

\section{Urteilswirkungen aufgrund der EMRK}

\section{a) Rechtskraft inter partes und einzelfallbezogene Befolgungspflicht (Art. 46 EMRK)}

\section{4}

Artikel 46 Abs. 1 EMRK lautet: „Die Hohen Vertragsparteien verpflichten sich, in allen Rechtssachen, in denen sie Partei sind, das endgültige Urteil des Gerichtshofs zu befolgen." Die hier genannte Befolgungspflicht gilt für den ,,verurteilten“ Staat, also inter partes. Wann ein Urteil „,endgültig“ wird, ergibt sich aus Art. 44 EMRK (z.B. alle Urteile der Großen Kammer). Endgültigkeit meint Rechtskraft: ${ }^{149}$ Endgültige Urteile können nicht mehr angefochten werden (formelle Rechtskraft). Des Weiteren sind diese Urteile auch inhaltlich für die Parteien verbindlich (materielle Rechtskraft). Die Vertragsstaaten können nicht mehr be-

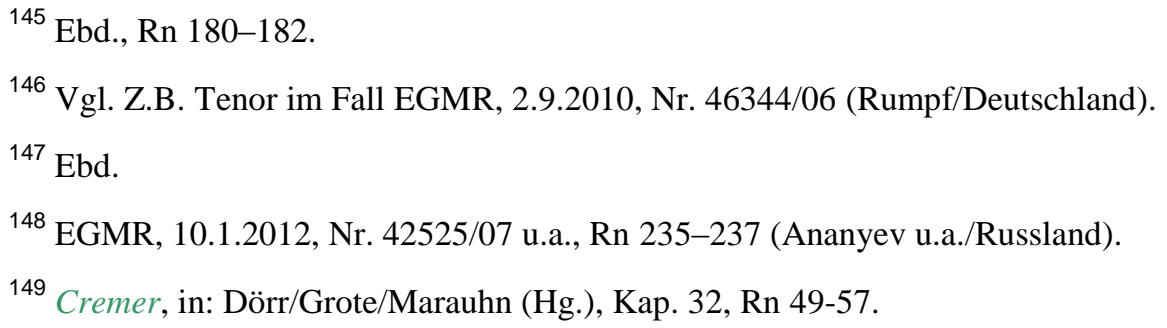


Anne Peters/Tilmann Altwicker, in: Jörg Philipp Terhechte (Hrsg.), Enyzklopädie des Europarechts, Band 3 (Baden-Baden: Nomos 2014)

haupten, ihr Verhalten sei konventionskonform gewesen. Die Rechtskraft bezieht sich allein auf den Tenor (die vom Gerichtshof benannten Rechtsfolgen) der Entscheidung. Die materielle Rechtskraft ist durch die personellen, sachlichen und zeitlichen Grenzen des Streitgegenstandes begrenzt. Dieser kann sich bis zu einem erneuten innerstaatlichen Verfahren so entscheidend ändern, dass neuen Beschwerden die Rechtskraft unter Umständen nicht entgegen steht.

Die Befolgungspflicht kann näher unter Rückgriff auf das Recht der Staatenverantwortlichkeit bestimmt werden: Der Staat muss eine andauernde Rechtsverletzung beenden (Beendigungsgebot). ${ }^{150}$ Wie der Vertragsstaat eine Konventionsverletzung beendet (z.B. durch Gesetzesänderung, konventionskonforme Auslegung oder durch eine individuelle Maßnahme), bleibt ihm grundsätzlich selbst überlassen. ${ }^{151}$ Der Vertragsstaat hat also ein Auswahlermessen, wie er seine völkerrechtlichen Verpflichtungen aus dem Urteil erfüllt. In Ausnahmefällen hat der EGMR aber dieses Auswahlermessen ,auf Null“ reduziert. ${ }^{152}$ Ferner trifft den Staat nach allgemeinem Völkerrecht die Pflicht, den durch die Konventionsverletzung entstandenen Schaden wiedergutzumachen (Wiedergutmachungsgebot). ${ }^{153}$ Dazu gehört vorrangig die weitestgehende Wiederherstellung des konventionsgemäßen Zustands (restitutio in integrum). ${ }^{154}$ Auch Art. 41 EMRK geht davon aus, dass vorrangig die Verletzung wieder gut gemacht werden muss (siehe im Einzelnen oben). ${ }^{155}$ Der Vertragsstaat muss also alles Zumutbare unternehmen, um den konventionsgemäßen Zustand im Einzelfall wiederherzustellen. Wenn dies nicht möglich ist, muss er nachrangig die oben angesprochene finanzielle Entschädigung leisten, deren Höhe vom EGMR festgesetzt wird. ${ }^{156}$ Bisweilen stellt bereits die Feststellung der Konventionsverletzung eine ausreichende Wiedergutmachung dar. ${ }^{157}$

\section{b) Allgemeine Pflicht des betroffenen Staates zur Korrektur der Rechtsordnung}

Die Befolgungspflicht trifft nach Art. 46 Abs. 1 ausdrücklich nur den Staat, der Partei des Verfahrens war Die Urteile entfalten formal keine erga omnes-Wirkung, sondern gelten - auf Seiten des Beschwerdegegeners und des Beschwerdeführers - inter partes. Daher können Drittpersonen, selbst wenn sie der Hoheitsgewalt des die Konvention ,verurteilten“ Staates unterworfen sind, grundsätzlich keine Rechte aus einem Urteil, das in einem anderen Verfahren gegen diesen Staat erging, herleiten. Dennoch muss der Staat, dessen

\footnotetext{
${ }^{150}$ Vgl. Art. 30 lit. a) ILC-Artikel (Fn. 64).

${ }^{151}$ EGMR, 17.9.2009, Nr. 10249/03, Rn 147 (Scoppola/Italien (Nr. 2)), NJOZ 2010, 2726.

152 EGMR, 8.4.2004, Nr. 71503/01 (Assanidze/Georgien).

${ }^{153}$ Vgl. Art. 31, 34 ILC-Artikel (Fn. 60 64) zu ,reparation“.

${ }^{154}$ Die Grundlage für die explizite Anordnung einer Naturalrestitution ist das allgemeine Völkerrecht (vgl. Art. 35 f. ILC-Artikel (Fn. 64) zur Naturalrestitution).

${ }^{155}$ Art. 41 gibt dem EGMR die Kompetenz zur Anordnung finanzieller Entschädigung, wenn das innerstaatliche Recht der Vertragspartei nur eine ,unvollkommene Wiedergutmachung“ gestattet.

${ }^{156}$ EGMR, 7.12.2010, Nr. 35720/04 u.a., Rn 28 (Vrioni u.a./Albanien).

${ }^{157}$ Dies ist der „Genugtuung“ (satisfaction) gem. Art. 37 ILC-Artikel (Fn. 64) vergleichbar.
} 
Anne Peters/Tilmann Altwicker, in: Jörg Philipp Terhechte (Hrsg.), Enyzklopädie des Europarechts, Band 3 (Baden-Baden: Nomos 2014)

Konventionsverletzung festgestellt wurde, seine Rechtsordnung mit der Konvention in Einklang bringen, um weitere gleichartige Rechtsverletzungen (in Bezug auf weitere Personen) zu verhindern. Diese Anpassungspflicht ergibt sich nicht aus der Rechtskraft, sondern kann - als Manifestation der Befolgungspflicht aus dem allgemeinen Recht der Staatenverantwortung, das ein Wiederholungsverbot statuiert, ${ }^{158}$ abgeleitet werden.

Die Befolgungspflicht gemäß Art. 46 EMRK - und damit das Beendigungs-, Nichtwiederholungs- und Wiedergutmachungsgebot - gilt für den Staat als Ganzen, d.h. die Pflichten richten sich an alle drei Staatsgewalten (Legislative, Exekutive, Judikative). Diese Pflicht bedeutet für den innerstaatlichen Richter, dass er - auch und gerade, so lange der Gesetzgeber eine konventionswidrige Rechtsnorm nicht aufgehoben hat - der Konvention im Einzelfall die volle Wirksamkeit verschaffen muss. Das bedeutet, dass er sich in erster Linie um eine konventionskonforme Auslegung des innerstaatlichen Gesetzes bemühen muss. Ist diese nicht möglich, muss der Richter innerstaatliches konventionswidriges Recht unangewendet lassen. ${ }^{159}$ Das wird damit begründet, dass die EMRK integraler Bestandteil der nationalen Rechtsordnungen geworden ist. Allerdings schreibt die Konvention nicht vor, welche innerstaatliche Instanz die Prüfung der Konventionskonformität vorzunehmen hat bzw. welche Instanz über die Nichtanwendung innerstaatlichen Rechts befinden darf. Es reicht daher aus, wenn oberste Gerichte (in Deutschland das BVerfG) diese Fragen im Rahmen einer Richtervorlage entscheiden. ${ }^{160}$

\section{c) Orientierungswirkung für andere Staaten}

58

Andere Konventionsstaaten sind nicht direkt aus dem Urteil verpflichtet. Der EGMR ist nicht formal an seine Präjudizien gebunden. Jedoch ist eine ,gefestigte Rechtsprechung“ rechtlich relevant, wie Art. 28 EMRK zeigt. Nach dieser Vorschrift kann eine Beschwerde schon durch einen Richterausschuss für begründet oder unbegründet erklärt werden, wenn die der Rechtssache zugrunde liegende Frage bereits Gegenstand einer gefestigten Rechtsprechung ist. Das BVerfG hat diese Relevanz als ,zumindest faktische

\footnotetext{
${ }^{158}$ Vgl. Art. 30 lit. b) ILC-Artikel (Fn. 64). Siehe zur Befolgungspflicht als Konsequenz der internationalen Staatenverantwortung oben Rn. 55.

${ }^{159}$ EGMR, 26.4.2007, Nr. 71525/01, Rn 103 (Popescu/Rumänien Nr. 2).

${ }^{160}$ Nach neuer Auffassung müssen deutsche Fachgerichte, die von der Konventionswidrigkeit eines entscheidungserheblichen Gesetzes überzeugt sind, nach Art. 100 GG eine Entscheidung des BVerfG einholen. Art. $100 \mathrm{GG}$ sollte teleologisch so ausgelegt werden, dass die hier geregelte Vorlagepflicht auch diese Situation umfasst, weil die Fachgerichte ansonsten, bei Missachtung der Konventionswidrigkeit, ihre verfassungsrechtliche Berücksichtigungspflicht bzw. das thematisch einschlägige Grundrecht in Verbindung mit dem Rechtsstaatsprinzip verletzen würden. So Payandeh, 388; anders noch Peters/Altwicker, EMRK, § 37 Rn 20.
} 
Anne Peters/Tilmann Altwicker, in: Jörg Philipp Terhechte (Hrsg.), Enyzklopädie des Europarechts, Band 3 (Baden-Baden: Nomos 2014)

Präzedenzwirkung“ bezeichnet. ${ }^{161}$ Aus dieser Sicht entfalten die Urteile eine ,jedenfalls faktische Orientierungs- und Leitfunktion. “'162

\section{Folgen im deutschen Recht}

\section{a) Innerstaatliche Folgen der Konventionswidrigkeit}

\section{9}

Die deutschen Gerichte und Behörden sind kraft Verfassungsrechts verpflichtet, deutsche Gesetze und Verordnungen konventionskonform (und unter Berücksichtigung der EGMR-Rechtsprechung, dazu sogleich unten) auszulegen. Prozessual stellt sich das Problem konventionswidriger Gesetze normalerweise in Klagen oder in einer Verfassungsbeschwerde gegen den Anwendungsakt mit Inzidentprüfung des Gesetzes. Ist eine konventionskonforme Auslegung nicht möglich, müssen deutsche Gerichte die Entscheidung des BVerfG gem. Art. 100 Abs. 1 GG einholen (s. Rn 57). Es ist gegenwärtig unklar, ob Gerichte in Deutschland eine bewusste Abweichung des Gesetzgebers von der EMRK respektieren müssten. ${ }^{163}$ In diesem Fall gälte im Innenverhältnis das Gesetz, wenn es die lex posterior zur EMRK ist. Im Außenverhältnis würde sich jedoch die Bundesrepublik wegen einer Völkerrechtsverletzung rechtlich verantwortlich machen. Um die völkerrechtliche Haftung zu vermeiden, sind unter Umständen Gesetzesänderungen erforderlich. Jüngstes Beispiel für eine Gesetzesänderung aufgrund der EGMR-Rechtsprechung ist die deutsche Neuregelung der Sicherungsverwahrung.

\section{0}

Ein konventionswidriger Verwaltungsakt ist ex tunc rechtswidrig und kann nach $\S 48(\mathrm{~L}) \mathrm{VwVfG}$ zurückgenommen werden. Nach hier vertretener Ansicht ist das Rücknahmeermessen in diesen Fällen,,auf Null“" reduziert, sodass die Behörde den rechtswidrigen Verwaltungsakt aufheben muss.

\section{1}

Die EMRK selbst verlangt grundsätzlich nicht die Aufhebung konventionswidriger Gerichtsurteile. Grund ist, dass die Konvention das Prinzip der Rechtskraft akzeptiert. Die entgegenstehende Rechtskraft ist die typische Situation, auf welche die Vorschrift des Art. 41 EMRK zugeschnitten ist: Bei Rechtskraft eines nationalen Urteils besteht nur eine unvollkommene Möglichkeit zur innerstaatlichen Wiedergutmachung, und genau dann kann der EGMR eine gerechte Entschädigung anordnen. In Deutschland ist das Problem konventionswidriger Gerichtsurteile dadurch entschärft worden, dass umfassende Wiederaufnahmetatbestände geschaffen wurden: So liegt ein Wiederaufnahmegrund für das Strafverfahren nach $§ 359$ Nr. 6 StPO vor, wenn der EGMR eine Verletzung der EMRK oder ihrer Protokolle festgestellt hat und das Urteil auf dieser Verletzung beruht. Darüber hinaus wurde mit Wirkung zum 1.1.2007 die EMRK-bezogene Restitutionsklage nach $\S 580$ Nr. 8 ZPO eingeführt. Auf diese Vorschrift verweisen die Normen in $\S 79$ ArbGG;

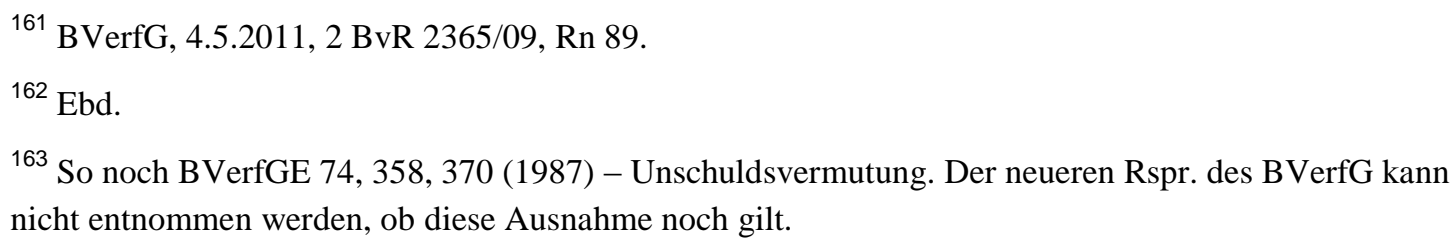


Anne Peters/Tilmann Altwicker, in: Jörg Philipp Terhechte (Hrsg.), Enyzklopädie des Europarechts, Band 3 (Baden-Baden: Nomos 2014)

179 SGG; 153 VwGO; 134 FGO. Nach diesen Vorschriften findet die Restitutionsklage statt, ,,wenn der EGMR eine Verletzung der EMRK festgestellt hat und das Urteil auf dieser Verletzung beruht.“

\section{b) Berücksichtigungspflicht}

\section{2}

Grundsätzlich gilt nach ständiger Rechtsprechung des BVerfG, dass die EMRK in Form der Auslegung durch den EGMR „,berücksichtigt“ werden muss. ${ }^{164}$ Die Berücksichtigungspflicht führt z.B. dazu, dass sich nationale Rechtsanwender mit den Entscheidungsgründen des EGMR auseinandersetzen müssen. ${ }^{165}$ Wenn sie von einem EGMR-Urteil abweichen wollen, müssen sie dies nachvollziehbar begründen. Diese Berücksichtigungspflicht erstreckt sich zunächst auf alle Entscheidungen des EGMR über denselben Streitgegenstand. Das BVerfG zieht aber auch Entscheidungen über andere Streitgegenstände (also solche, die von anderen Beschwerdeführern ausgingen, und solche gegen andere Staaten als Beschwerdegegner) heran. ${ }^{166}$ Das heißt insbesondere, dass auch ,nichtdeutsche“ Fälle ${ }^{167}$ berücksichtigt werden müssen. Die verfassungsgestützte Pflicht zur konventionskonformen Auslegung und zur Berücksichtigung hat allerdings eine Grenze: Diese ist nach dem BVerfG erreicht, wo die Anwendung der EMRK entweder in Konflikt mit einem klar anderslautenden Willen des Gesetzgebers oder mit deutschem Verfassungsrecht gerät. Ein automatischer Vorrang der EMRK vor anderem Bundesrecht wird vom BVerfG ausdrücklich ausgeschlossen. ${ }^{168}$

\section{c) Verfassungsbeschwerde mit Durchsetzungsrüge}

\section{3}

Da es sich bei den EMRK-Rechten nicht um Grundrechte des GG handelt, kann eine Verfassungsbeschwerde nicht auf die bloße Verletzung von Konventionsrechten gestützt werden (vgl. Art. 93 Abs. 1 Nr. 4a GG). Wegen der besonderen Verantwortung des BVerfG für die Durchsetzung des Völkerrechts in Deutschland hat aber das BVerfG die Möglichkeit einer Durchsetzungsrüge geschaffen: So kann eine Verfassungsbeschwerde erhoben werden, wenn ein Staatsorgan gegen die Berücksichtigungspflicht verstoßen hat. Der Beschwerdeführer kann hier rügen, dass die Nichtberücksichtigung der EMRK, und vor allem eines die EMRK konkretisierenden Urteils des EGMR, das materiell betroffene, thematisch einschlägige Grundrecht des GG in Verbindung mit dem Rechtsstaatsprinzip verletze. ${ }^{169}$

${ }^{164}$ Diese wird begründet mit der Völkerrechtsfreundlichkeit des GG und mit der Erwägung, dass völkerrechtliche Verurteilungen der Bundesrepublik Deutschland vermieden werden sollen.

${ }^{165}$ BVerfGE 111, 307, 324 (2004) - Görgülü; BVerfG, 26.6.2009, 1 BvR 134/03, Rn 69.

${ }^{166}$ BVerfG, 4.5.2011, 2 BvR 2365/09, Rn 89 f.

${ }^{167}$ So die Formulierung von Karpenstein/Mayer/Breuer, EMRK, Art. 46, Rn 54.

${ }^{168}$ BVerfGE 111, 307, 329 (2004) - Görgülü.

${ }^{169}$ Ebd., 329 f. 
Anne Peters/Tilmann Altwicker, in: Jörg Philipp Terhechte (Hrsg.), Enyzklopädie des Europarechts, Band 3 (Baden-Baden: Nomos 2014)

\section{Urteilsdurchsetzung}

64

Weit überwiegend werden die Urteile des EGMR problemlos befolgt. Darüber hinaus zahlen ca. 80 Prozent der verurteilten Staaten die Entschädigung innerhalb der Jahresfrist. Das Ministerkomitee (Organ des Europarates nach Art. 10; 13 ff. der Satzung des Europarates) ist zuständig für die Überwachung der Befolgung der Urteile des EGMR (Art. 46 Abs. 2 EMRK). Das Verfahren der Urteilsdurchsetzung wurde zunehmend verrechtlicht. So verlangen die seit 2006 geltenden „Regeln des Ministerkomitees zur Überwachung der Urteilsdurchsetzung“ (RÜD) neben der Überprüfung, ob eine gerechte Entschädigung gezahlt wurde (Regel 6 Abs. 2 lit. a RÜD), auch die Berücksichtigung etwaiger vom Staat getroffener individueller oder gesetzlicher Maßnahmen (Regel 6 Abs. lit. b RÜD). ${ }^{170}$ Kommt das Ministerkomitee zu dem Ergebnis, dass der Vertragsstaat alle Verpflichtungen aus dem Urteil erfüllt hat, schließt es das Überwachungsverfahren mit einer „final resolution“ ab (Regel 17 RÜD). Hält das Ministerkomitee dagegen die Urteilsdurchführung durch den Vertragsstaat für unzureichend, kann es durch eine „,interim resolution“ (Regel 16 RÜD) politischen Druck ausüben. Außerdem wurde mit dem 14. Änderungsprotokoll ein neues Urteilsbefolgungsverfahren eingeführt: Das Ministerkomitee kann nun den EGMR mit der Frage befassen, ob der Staat seiner Urteilsbefolgungspflicht nach Art. 46 Abs. 1 EMRK nachgekommen ist (Art. 46 Abs. 4, 5 EMRK). Der Gerichtshof wird dann in einem zweiten Urteil feststellen, ob der Staat durch Nichtbefolgung des Ersturteils Art. 46 Abs. 1 EMRK verletzt hat. Die Frage der ursprünglichen Vertragsverletzung wird damit nicht noch einmal aufgeworfen, und es ist auch keine (zusätzliche) Entschädigung für die Nichtbefolgung vorgesehen. Die Autoren des 14. Änderungsprotokolls erwarten, dass das Ministerkomitee dieses Verfahren nur sehr selten anstoßen wird, dass aber die bloße Möglichkeit einen weiteren Anreiz für die Urteilsbefolgung schafft. In den drei Jahren seit seiner Einführung ist noch kein Urteilsbefolgungsverfahren eingeleitet worden.

\section{Ausblick}

65

Im Verfahrensrecht des EGMR sind vier Tendenzen auszumachen: Erstens müssen die Bemühungen der Effizienzsteigerung des Konventionsrechtsschutzsystems fortgeführt und intensiviert werden. ${ }^{171}$ Dies ist notwendig, weil sich seit 2004 die Zahl der jährlich anhängig gemachten Beschwerden in jedem Jahr verdoppelt hat, sodass Beschwerdeführer heute ca. vier Jahre auf eine Entscheidung des EGMR warten müssen. Das Inkrafttreten des 14. Prot. hat mit der Einführung der Einzelrichterentscheidung, den erweiterten Befugnissen der Ausschüsse (Art. 28 Abs. 1 lit. b) EMRK) und dem neuen Unzulässigkeitskriterium des fehlenden erheblichen Nachteils (Art. 35 Abs. 3 lit. b) EMRK) hier erste Fortschritte gebracht. Diese Maßnahmen beziehen sich vor allem auf die vielen Wiederholungsfälle und „leichte“ Konventionsverletzungen.

\footnotetext{
${ }^{170}$ Rules of the Committee of Ministers for the supervision of the execution of judgments and of the terms of friendly settlements v. 10.5.2006.

${ }^{171}$ Siehe hierzu die Vorschläge der High Level Conference on the Future of the European Court of Human Rights, Brighton Declaration vom 20.4.2012, Rn 16. Angestrebt werden u.a. eine Verbesserung des Konventionsrechtsschutzes auf nationaler Ebene, eine Weiterentwicklung des Piloturteilsverfahrens in Richtung einer Art „class action” und eine Entfernung der mit dem 14. Prot. neu eingeführten Rechtsverweigerungsklausel (hierzu Rn 23, dort auch zur Umsetzung im noch nicht in Kraft getretenen 15. Prot.). Zur Brighton Declaration s. Tomuschat, Brighton.
} 
Anne Peters/Tilmann Altwicker, in: Jörg Philipp Terhechte (Hrsg.), Enyzklopädie des Europarechts, Band 3 (Baden-Baden: Nomos 2014)

Andere Mechanismen wurden eingeführt, um die ebenfalls zu einer Vielzahl von Beschwerden führenden systemischen Probleme in den Vertragsstaaten angehen zu können. Hier sind vor allem das Piloturteilsverfahren und das Vorrangverfahren nach Regel 41 S. 2 EGMR-VerfO, mit dem der Gerichtshof die Behandlung einer Beschwerde vorziehen kann, zu erwähnen.

66

Zweitens wird der Beitritt der EU zur EMRK (Art. 6 Abs. 2 S. 1 EUV i.d.F. des Lissabonner Vertrags) wichtige materielle und prozedurale Änderungen mit sich bringen. ${ }^{172}$ Die Konvention wird Bestandteil der Unionsrechtsordnung. Im Rang werden die Konventionsrechte - wenn sie als normales Völkervertragsrecht angesehen werden - unterhalb des EU-Primärrechts stehen, aber mit Vorrang vor dem Sekundärrecht ausgestattet sein (vgl. Art. 216 Abs. 2 AEUV). Das heißt, dass alle EU-Akte materiell konventionskonform ausgestaltet werden müssen. Neben den Gerichten der Mitgliedstaaten müssen dann auch die Gerichte der EU die EMRK als Maßstab der Rechtsprüfung anwenden (letztere z.B. bei Nichtigkeitsklagen gegen EUAkte). Einzelne können dann beim EGMR Beschwerde mit der Rüge erheben, dass ein Unionsakt sie in einem ihrer Konventionsrechte verletzt. Artikel 36 Abs. 4 EMRK n.F. ${ }^{173}$ ermöglicht ein gemeinsames Beschwerdeverfahren (,co-respondent mechanism“) gegen die EU und involvierte Mitgliedstaaten.

\section{7}

Drittens hält die Diskussion um die Fortentwicklung des EGMR zu einem europäischen Verfassungsgericht an. ${ }^{174}$ Hiermit ist gemeint, dass der EGMR nicht primär als Instanz für die Beseitigung von Menschenrechtsverletzungen im Einzelfall fungieren soll, sondern primär als Institution, deren Hauptziel es ist „das allgemeine Niveau des Menschenrechtsschutzes anzuheben“, eine „öffentliche Ordnung“ Europas zu schaffen und die strukturellen Probleme zu verdeutlichen, welche die Demokratie und den Rechtsstaat in Europa untergraben. ${ }^{175}$ Um diese Rolle auszufüllen, müsste sich der Gerichtshof auf wenige richtungsweisende Grundsatzentscheidungen konzentrieren können. Der Verfassungsgerichts-Vorschlag ist einerseits eine Reaktion auf die Überlastung des Gerichtshofs, beinhaltet daneben jedoch einen grundsätzlichen Perspektivenwechsel. Bisherige Reformen (Piloturteilsverfahren und Einführung der Zulässigkeitsvoraussetzung des ,erheblichen Nachteils“) und auch das von der Brighton-Erklärung vorgeschlagene Gutachtenverfahren ${ }^{176}$ gehen in eine „,verfasssungsgerichtliche“ Richtung. Die Qualifikation der EMRK als „Verfassungsinstrument“ (constitutional instrument) durch den EGMR selbst unterstreicht diese

\footnotetext{
${ }^{172}$ Vgl. die Darstellung bei Grewe.

${ }^{173}$ Draft Agreement on the Accession of the EU to the Convention, 25.2.2011, CDDH-UE(2011)04. Hierzu Draft Explanatory Report to the Agreement on the Accession of the European Union to the Convention for the Protection of Human Rights and Fundamental Freedoms, 2.5.2011, CDDH-UE(2011)08, Rn 29 ff.

${ }^{174}$ Hierzu grundlegend Wildhaber. Die Eigenschaft des EGMR als „Verfassungsgericht“" Europas bejaht Häberle, 479 f. Vgl. auch Stone Sweet.

${ }^{175}$ Wildhaber (Fn. 174), 571 u. 573.

${ }^{176}$ Brighton Declaration (Fn. 171), Rn 12 d).
} 
Anne Peters/Tilmann Altwicker, in: Jörg Philipp Terhechte (Hrsg.), Enyzklopädie des Europarechts, Band 3 (Baden-Baden: Nomos 2014)

Tendenz ebenfalls. ${ }^{177}$ Die weitergehende Forderung nach einem Annahmeermessen des Gerichtshofs (in der Art des Certiorari-Verfahrens des U.S.-Supreme Courts) hat sich jedoch bisher nicht durchgesetzt.

\section{8}

Eine vierte Tendenz zeigt sich an der verstärkten Kritik am EGMR unter Hinweis auf Legitimationsdefizite der Rechtsprechung. In der Tat birgt richterlicher Aktivismus in Form der dynamischen Auslegung der Konvention, die teilweise an Rechtsschöpfung grenzt, die Gefahr der Unterminierung der mitgliedstaatlichen Akzeptanz des Gerichtshofs und der Konterkarierung rechtspolitischer Entscheidungen, die in den Mitgliedstaaten auf der Grundlage eines demokratischen Prozesses gefällt wurden. In Reaktion auf Urteile, die im Widerspruch zu nationalen Traditionen stehen, wurde neuerdings eine Möglichkeit der „Überstimmung“ („democratic override“) Straßburger Entscheidungen durch innerstaatliche Institutionen verlangt. ${ }^{178}$ $\mathrm{Zu}$ Recht hat sich diese Forderung bislang nicht durchsetzen können: Zum einen würde die konventionsrechtlich vorgesehene Funktionsteilung zwischen Straßburg und den nationalen Stellen aus dem Gleichgewicht gebracht, zum anderen würden hierdurch die gleichmäßige Anwendung und letztlich die Effektivität des konventionsrechtlichen Rechtsschutzes insgesamt beschädigt. Grundsätzlich erscheint der Gerichtshof ausreichend legitimiert. Elemente wie die Öffentlichkeit des Gerichtsverfahrens und die Möglichkeit der Drittbeteiligung tragen zu seiner institutionellen und prozeduralen Legitimation bei. Die Beachtung des Einschätzungsspielraums der Mitgliedstaaten (margin of appreciation/marge d'appréciation) insbesondere in Fragen, die moralisch aufgeladen sind und in denen sich noch kein gesamteuropäischer Konsens gebildet hat, ${ }^{179}$ dient der materiellen und sozialen Legitimation der Entscheidungen. Die Brighton-Erklärung der Konferenz der Mitgliedstaaten von April 2012 und das auf dieser Linie liegende 15. Protokoll (noch nicht in Kraft) präsentieren vor allem stärkere Beachtung des Subsidiaritätsprinzips durch den Gerichthof selbst gegenüber den Mitgliedstaaten als Abhilfe gegen die wahrgenommenen Legitimationsprobleme. ${ }^{180}$

${ }^{177}$ Der Begriff „,constitutional instrument“ wurde erstmals vom Richter Sir Gerald Fitzmaurice in seiner Sondermeinung im Fall EGMR, 27.10.1975 (National Union of Belgian Police/Belgien), Nr. 4464/70, Annex Rn 9 gebraucht = EuGRZ 1975, 562. Vgl. auch EGMR, 23.3.1995, Nr. 15318/89 (Loizidou Nr. 1/Türkei), ÖJZ 1995, 629; zuletzt EGMR 25.4.2013, Nr. 71386/10 (Dzhurayev/Russland), Rn 213.

${ }^{178}$ Vgl. Raab, Strasbourg in the Dock: Prisoner Voting, Human Rights and the Case for Democracy, London 2011. Auslöser dieser Forderung waren Urteile des EGMR, in denen dieser die traditionelle britische Aberkennung des Wahlrechts für Häftlinge als Verletzung von Art. 3 Zusatzprot. I qualifizierte (EGMR, 23. 11.2010, Nr. 60041/08 und 60054/08 (Greens/VK) und Folgeentscheidungen). In Russland wurde die Rechtsprechung zur Benachteiligung männlicher Militärangehöriger im Hinblick auf Erziehungsurlaub kritisiert (EGMR, 7.10.2010, Nr. 30078/06 (Markin/Russland), GK 22.3.2012). Hierzu Anm. L. Maelksoo, AJIL 2012, 836 ff. In der Schweiz wurde ein Urteil zur Kostentragungspflicht bei geschlechtsumwandelnden Operationen (EGMR, 8.1.2009, Nr. 29002/06 (Schlumpf/Schweiz) vom Bundesgericht kritisiert (BGE 137 I 86 (2010); kritisch auch BGer., 30.08.2013, 2C_365/2013, E.2.4. zur EGMR-Rspr. zur Ausweisung eines straffälligen Ausländers).

${ }^{179}$ Hierzu Peters/Altwicker, EMRK, § 3, Rn $18 \mathrm{ff}$.

${ }^{180}$ Das 15. Protokoll (Fn. 57) sieht die Aufnahme des Subsidiaritätsprinzips in die Präambel der EMRK vor, um dessen Bedeutung zu betonen. Dies entspricht der Brighton Declaration (Fn. 171), die darüber hinaus die Aufnahme des Prinzips des margin of appreciation in die Präambel der Konvention forderte (s. z.B. Rn 3, 11, 12b). 
Anne Peters/Tilmann Altwicker, in: Jörg Philipp Terhechte (Hrsg.), Enyzklopädie des Europarechts, Band 3 (Baden-Baden: Nomos 2014)

69

Verzeichnis wichtiger Entscheidungen

\begin{tabular}{|c|c|c|c|c|c|}
\hline Gericht & Datum & $\mathrm{Az}$ & Sammlung & Benennung & Fundstellen \\
\hline BVerfG & 14.10.2004 & $\begin{array}{l}2 \mathrm{BvR} \\
1481 / 04\end{array}$ & E 111,307 & Görgülü & $\begin{array}{l}\text { NJW 2004, } \\
3407\end{array}$ \\
\hline BVerfG & 4.5.2011 & $\begin{array}{l}2 \\
\text { BvR2365/09 }\end{array}$ & E 128,326 & Sicherungsverwahrung & $\begin{array}{l}\text { NJW 2011, } \\
1931\end{array}$ \\
\hline EGMR & 9.10 .1979 & Nr. 6289/73 & & Airey/Irland & $\begin{array}{l}\text { EuGRZ } \\
1979,626\end{array}$ \\
\hline EGMR & 7.8.1989 & $\begin{array}{l}\text { Nr. } \\
14038 / 88\end{array}$ & & $\begin{array}{l}\text { Soering/Vereinigtes } \\
\text { Königreich }\end{array}$ & $\begin{array}{l}\text { EuGRZ } \\
1989,314= \\
\text { NJW 1990, } \\
2183\end{array}$ \\
\hline EGMR & 23.3.1995 & $\begin{array}{l}\text { Nr. } \\
15318 / 89\end{array}$ & & Loizidou/Türkei & $\begin{array}{l}\text { ÖJZ } 1995, \\
629\end{array}$ \\
\hline EGMR & 5.4 .2000 & $\begin{array}{l}\text { Nr. } \\
34382 / 97\end{array}$ & & Dänemark/Türkei & $\begin{array}{l}\text { EuGRZ } \\
2000,619\end{array}$ \\
\hline EGMR & 12.12.2001 & $\begin{array}{l}\text { Nr. } \\
52207 / 99\end{array}$ & & $\begin{array}{l}\text { Banković u. a./17 Mit- } \\
\text { gliedstaaten }\end{array}$ & $\begin{array}{l}\text { NJW 2003, } \\
413\end{array}$ \\
\hline EGMR & 8.4 .2004 & $\begin{array}{l}\mathrm{Nr} . \\
71503 / 01\end{array}$ & & Assanidze/Georgien & $\begin{array}{l}\text { EuGRZ } \\
2004,268= \\
\text { NJW 2005, } \\
2207\end{array}$ \\
\hline EGMR & 22.4 .2004 & $\begin{array}{l}\text { Nr. } \\
31443 / 96\end{array}$ & & Broniowski/Polen & $\begin{array}{l}\text { NJW 2005, } \\
2521\end{array}$ \\
\hline EGMR & 8.7.2004 & $\begin{array}{l}\text { Nr. } \\
48787 / 99\end{array}$ & & $\begin{array}{l}\text { Ilaşcu u.a./Moldawien } \\
\text { u. Russland }\end{array}$ & $\begin{array}{l}\text { NJW 2005, } \\
1849\end{array}$ \\
\hline EGMR & 16.11.2004 & $\begin{array}{l}\text { Nr. } \\
31821 / 96\end{array}$ & & Issa/Türkei & \\
\hline EGMR & 4.2.2005 & $\begin{array}{l}\text { Nr. } \\
46827 / 99 \\
\text { u.a. }\end{array}$ & & $\begin{array}{l}\text { Mamatkulov } \\
\text { u.a./Türkei }\end{array}$ & $\begin{array}{l}\text { EuGRZ } \\
2005,357\end{array}$ \\
\hline EGMR & 12.5 .2005 & Nr. & & Öcalan/Türkei & EuGRZ \\
\hline
\end{tabular}


Anne Peters/Tilmann Altwicker, in: Jörg Philipp Terhechte (Hrsg.), Enyzklopädie des Europarechts, Band 3 (Baden-Baden: Nomos 2014)

\begin{tabular}{|c|c|c|c|c|c|}
\hline Gericht & Datum & $\mathrm{Az}$ & Sammlung & Benennung & Fundstellen \\
\hline & & $46221 / 99$ & & & $\begin{array}{l}2005,463= \\
\text { NVwZ } \\
2006,1267\end{array}$ \\
\hline EGMR & 6.7 .2005 & $\begin{array}{l}\text { Nr. } \\
43577 / 98 \\
\text { u.a. }\end{array}$ & & $\begin{array}{l}\text { Nachova } \\
\text { u.a./Bulgarien }\end{array}$ & $\begin{array}{l}\text { EuGRZ } \\
2005,693\end{array}$ \\
\hline EGMR & 8.3.2006 & $\begin{array}{l}\text { Nr. } \\
59532 / 00\end{array}$ & & Blečić/Kroatien & $\begin{array}{l}\text { NJW 2007, } \\
347\end{array}$ \\
\hline EGMR & 9.9.2008 & $\begin{array}{l}\text { Nr. } \\
73250 / 01\end{array}$ & & $\begin{array}{l}\text { Boivin/34 Mitglied- } \\
\text { staaten d. Europarats }\end{array}$ & \\
\hline EGMR & 29.3.2010 & Nr. 3394/03 & & $\begin{array}{l}\text { Medvedyev } \\
\text { u.a./Frankreich }\end{array}$ & $\begin{array}{l}\text { NJOZ 2011, } \\
231\end{array}$ \\
\hline EGMR & 1.7.2010 & $\begin{array}{l}\text { Nr. } \\
25551 / 05\end{array}$ & & Korolev/Russland & $\begin{array}{l}\text { NJW 2010, } \\
3081\end{array}$ \\
\hline EGMR & 7.7.2011 & $\begin{array}{l}\text { Nr. } \\
55721 / 07\end{array}$ & & $\begin{array}{l}\text { Al-Skeini } \\
\text { u.a./Vereinigtes Kö- } \\
\text { nigreich }\end{array}$ & $\begin{array}{l}\text { NJW 2012, } \\
283\end{array}$ \\
\hline EGMR & 13.12 .2011 & $\begin{array}{l}\text { Nr. } \\
38263 / 08\end{array}$ & & $\begin{array}{l}\text { Georgien/Russland Nr. } \\
2\end{array}$ & \\
\hline EGMR & 23.2.2012 & $\begin{array}{l}\mathrm{Nr} . \\
27765 / 09\end{array}$ & & Hirsi Jamaa u.a./Italien & $\begin{array}{l}\text { NVwZ } \\
2012,809\end{array}$ \\
\hline EGMR & 15.3.2012 & $\begin{array}{l}\text { Nr. } 4149 / 04 \\
\text { u.a. }\end{array}$ & & Aksu/Türkei & \\
\hline EGMR & 12.9.2012 & $\begin{array}{l}\mathrm{Nr} . \\
10593 / 08\end{array}$ & & Nada/Schweiz & $\begin{array}{l}\text { NJOZ 2013, } \\
1183\end{array}$ \\
\hline
\end{tabular}

